\title{
Mathematical Relationships of Linearized Modeling Methods of AC Power Electronic Systems
}

Yicheng Liao and Xiongfei Wang

This is a preprint of the paper submitted to IEEE Transactions on Power Electronics, which is still under review.

Citation of this work :

Yicheng Liao and Xiongfei Wang, "Mathematical Relationships of Linearized

Modeling Methods of AC Power Electronic Systems", IEEE TechRxiv, Jan. 2021, doi:10.36227/techrxiv.13634072.

Comments and suggestions are welcomed.

Contact information: Yicheng Liao,ycl@et.aau.dk, Xiongfei Wang,xwa@et.aau.dk

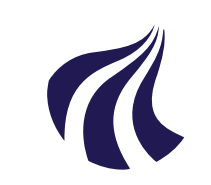

AALBORG UNIVERSITY

DENMARK

Copyright 2021 IEEE. Personal use of this material is permitted. Permission from IEEE must be obtained for all other uses, in any current or future media, including reprinting/republishing this material for advertising or promotional purposes, creating new collective works, for resale or redistribution to servers or lists, or reuse of any copyrighted component of this work in other works. 


\title{
Mathematical Relationships of Linearized Modeling Methods of AC Power Electronic Systems
}

\author{
Yicheng Liao, Student Member, IEEE, and Xiongfei Wang, Senior Member, IEEE
}

\begin{abstract}
The harmonic state-space, the dynamic phasor, and the generalized $d q$ modeling are three methods developed for linearization of ac power electronic systems. This paper reveals explicitly mathematical relationships between the three modeling methods in both time and frequency domain. Representations of linearized models in different reference frames and from time domain to frequency domain, as well as their transformations are elaborated step by step. Case studies on a three-phase voltagesource converter that is connected to an unbalanced grid verify the theoretical findings.
\end{abstract}

\section{INTRODUCTION}

AC power electronic systems are widely found in modern power grids, driven by the large-scale integration of renewable power generation. Their control dynamics tend to interact with the power grid and lead to control-interaction issues in a wide frequency range [1]. It is of paramount importance to model and analyze the dynamics of converter-based power systems. The linearized modeling methods are commonly used, as they allow the use of small-signal analysis based on eigenvalues or frequency domain tools.

To retain control dynamics of ac converter-based systems, the state-space averaging (SSA) over the switching period is generally applied [2], [3], which yields an averaged dynamic model that is essentially nonlinear and time-periodic. The conventional way to model such systems is to apply the Park transformation first to establish a time-invariant system in the synchronous reference $(d q)$ frame, and then the linearization around equilibrium points of the system can be conducted to obtain a linear time-invariant (LTI) model [4]. The $d q$-frame model can be represented with real vectors [5], [6] or complex vectors [7]-[10], and even be transformed back to the stationary reference $(\alpha \beta)$ frame [11], [12]. However, these models only apply to balanced three-phase systems. In the presence of unbalanced or harmonically distorted voltage, the time periodicity is also present in the $d q$ frame. Consequently, their dynamics cannot be characterized by the aforementioned models.

To characterize the time-periodic dynamics in unbalanced or harmonically distorted ac systems, three modeling methods are developed: 1) the harmonic state space (HSS) modeling [13]-[18]; 2) the dynamic phasor (DP) modeling [19]-[22]; 3) the generalized $d q$ (GDQ) modeling [23]-[25].

The HSS characterizes frequency responses of linear timeperiodic (LTP) systems [26], thus a prior linearization directly around the steady-state trajectories is required [27], [28]. The HSS model results in a harmonic transfer function (HTF) in the frequency domain, which is, essentially, an LTI transfer function matrix, revealing dynamic couplings between the
Fourier coefficients of harmonics [29]. The HSS modeling has been used for dynamic analyses of single-phase converters [13], modular multilevel converters [15], [16], and three-phase converters in unbalanced grids [17], [18]. While originally derived with real-valued LTP models, it is later found that the HSS model can also be used to represent complex-valued LTP models, which facilitates the integration of closed-loop control dynamics into power stages of converters [16], [17], [30].

The DP model is derived from the generalized averaging (GA) operator [31], [32]. Given a fixed system fundamental frequency, the GA operator calculates the Fourier coefficients of time-periodic variables over a moving time window [31]. The time-periodic system can thus be transformed into multiple time-invariant systems represented by differential equations of these Fourier coefficients. Then, the linearization around their equilibrium points can be performed [33]. This method has been widely applied to model power converters in unbalanced grid conditions [19], [22] or with multiple harmonics [20], [21]. The GA can be in general applied in any reference frame and to either real-valued or complex-valued variables [22].

The GDQ model is derived based on GDQ transformation [23], which similarly yields time-invariant systems in multiple $d q$ reference frames. The idea was initiated in [34] to model an unbalanced ac system. However, the multiple $d q$-frame model in [34] overlooked the couplings between different $d q$ frames, since the unbalanced system is still time-periodic in $d q$ frames. This flaw was addressed in [23] by invoking the principle of harmonic balance. The GDQ modeling is recently applied to multi-level modular converters [24], [25].

The three modeling methods have been developed based on different principles for a long time, until a few recent works exploring their relationships. The equivalence between the HSS model and DP model has been implicitly discussed in [35], since the DP model yields the same state-space matrices as the HSS model. Their relationships were further thoroughly studied in [33], [36], [37]. In [36], the DP model was claimed as less accurate than the HSS model, considering that the highfrequency dynamics was assumed to be neglected for the DP model. In [33], the linearization principles of the two methods were discussed, i.e., the HSS model is based on linearization around the time-periodic trajectories, whereas the DP model is based on linearization around the time-invariant points. In [37], the equivalence of two methods was proved by the eigenvalue analysis of the state-space matrices, through a comparative study on a converter system. For the GDQ modeling, its equivalence to applying the DP modeling in the $\alpha \beta$ frame was studied in [38] by proofs in the complex space. The equivalence of the GDQ model and HSS model was 
studied in [39] based on the principle of harmonic balance using Fourier series, and their relationships were explained by the complex transformation. However, these works merely apply GDQ transformations without considering the initial phase impacts. Such initial phase impacts cannot be avoided in multiple-converter systems, since the initial phases of their steady-state trajectories could be different [8], [12].

While the equivalences of these models have been proved by the eigenvalue analysis [37], [38] and been explained based on the harmonic balance [33], [39], there is still lack of a discussion on the common mathematical basis that leads to the model equivalence. Furthermore, the explicit mathematical relationships among the principles of these methods are still missing. This paper is thus dedicated to filling in these gaps by

- Clarifying the common mathematical bases of linearized modeling methods for ac power electronic systems. It is revealed that the system steady-state frequencies can determine an orthogonal basis for the time-invariant representation of ac systems.

- Unveiling mathematical relationships among principles of the HSS, the DP, and the GDQ modeling methods. It is found that the HSS (HTF) model can be derived from the DP model through a Laplace transformation, whereas the DP model can be transformed from the GDQ model through an initial phase rotation.

- Elaborating how different modeling methods eventually yield a unified stationary-frame HTF model, which can characterize the ac-side and dc-side frequency couplings for ac converter systems.

The rest of the paper is organized as follows. Section II reveals the common mathematical basis for the linearized modeling of ac systems. Section III derives the mathematical relationships of the HSS, the DP, and the GDQ modeling, which are verified on a three-phase voltage-source converter under unbalanced grid conditions in Section IV. Section V finally concludes the paper.

\section{MATHEMATICAL BASES}

The mathematical bases of different linearized modeling methods are reviewed in this section, including the definition of equilibria for ac systems, the transformations for model representations and their properties in relation to the linearization are introduced. Finally, the common mathematical basis of different modeling methods is summarized.

In the following derivations, variables without any subscript, e.g., $x(t)$, can be defined in any reference frame and can be either real-valued or complex-valued. If with the subscript " $\alpha \beta z$ " or " $d q z$ ", the variables are defined in the corresponding reference frame. Variables in bold letters, e.g., $\mathbf{x}(t)$, indicate the complex-valued variable representation. Variables with capital letters or the subscript " 0 " represent the equilibrium point or the steady-state trajectory. " $\Delta$ " before variables denotes the small-signal dynamics. $\mathbb{Z}$ denotes the integer set, $\mathbb{R}$ denotes the real number set, and $\mathbb{C}$ denotes the complex number set.

\section{A. Equilibria of AC Systems}

The small-signal model is derived based on the linearization around the equilibrium of a system. The equilibrium of a system is defined as $x_{0}(t)$, where $x$ can represent any variable in the system and can be either real or complex.

For a time-invariant system, its equilibrium stays at a fixed point [40], which satisfies

$$
x_{0}(t) \equiv X_{0} \in \mathbb{R} \text { or } \mathbb{C} \text { such that } \frac{\mathrm{d} x_{0}(t)}{\mathrm{d} t}=0 .
$$

For an ac system that is time $(T)$-periodic, where $T$ is the fundamental period, its equilibrium travels along a trajectory or orbit periodically [40], which satisfies

$$
x_{0}(t)=x_{0}(t+T) \text {. }
$$

A $T$-periodic dynamic system may have multiple frequency components of $k \cdot f_{\mathrm{s}}$, where $f_{\mathrm{s}}=1 / T\left(\omega_{\mathrm{s}}=2 \pi / T\right)$. Its steady-state trajectory can be presented based on Fourier series expansion:

$$
x_{0}(t)=\sum_{k} X_{k} e^{j k \omega_{s} t} \in \mathbb{R} \text { or } \mathbb{C} .
$$

This implies that the equilibria of a $T$-periodic system can be represented by the time-invariant Fourier coefficients, i.e., $\left[\begin{array}{lllll}\cdots & X_{-k} & \cdots & X_{k} & \cdots\end{array}\right]^{\mathrm{T}}$, with the set of exponential functions $\left\{k \in \mathbb{Z} \mid e^{j k \omega_{s} t}\right\}$ serving as an orthogonal basis [41]. Due to the orthogonality of the basis functions, these Fourier coefficients are linearly independent to each other. Consequently, any variables in a $T$-periodic system can be represented by the linear combination of their Fourier coefficients.

It is worth noting that the linearization does not necessarily require a time-invariant representation of the system. There are thus two ways of linearized modeling for ac systems. The first way is to apply a direct linearization around the $T$ periodic trajectory of an ac system, yielding an LTP system [27]. The HSS modeling follows this principle [26].

The second approach is to transform the ac system into a reference frame that yields a time-invariant representation of the system. Then, the system is linearized as an LTI model. This is the general idea of the DP modeling and the GDQ modeling. Transformations used for time-invariant representations are introduced in Part B, and the linearization is introduced in Part $C$. Then the common mathematical basis of different modeling methods is summarized in Part $D$.

\section{B. Transformations}

The averaging operator and DQ/GDQ transformations are commonly used to model ac systems as time-invariant systems. The complex transformation is recently used to further simplify the model representation and coordinate transformations [7].

In the following discussions, transformations are elaborated based on signals, yet some of them also apply to systems, provided that they are reversible, i.e.,

$$
y=G u \Rightarrow P y=P G u=P G P^{-1} P u .
$$

where $u$ and $y$ are the input and output, $G$ represents the system model, and $P$ represents the transformation. 


\section{1) Averaging Operators}

The averaging operator applies an integral operation within a time window $T$ to a $T$-periodic signal. There are two wellknown averaging techniques: i) the SSA given by

$$
\bar{x}(t)=\frac{1}{T} \int_{t-T}^{t} x(\tau) d \tau
$$

and ii) the GA given by

$$
\langle x\rangle_{k}(t)=\frac{1}{T} \int_{t-T}^{t} x(\tau) e^{-j k \omega_{s} \tau} d \tau=x_{k} \text { for } k \in \mathbb{Z} .
$$

The SSA only retains the dynamics less than $\omega_{s} / 2\left(\omega_{s}=2 \pi\right.$ $/ T)$, which is usually used to filter out the switching dynamics of converters by choosing $T$ as the switching period. It is noted that for ac converter systems, the SSA over a switching period still leads to a time-periodic system, whose period is related to the fundamental frequency of the ac system.

The GA calculates the $k$-th Fourier coefficient of the timeperiodic signal, which is also based on the Fourier series expansion, i.e.,

$$
x(t)=\sum_{k}\langle x\rangle_{k}(t) e^{j k \omega_{s} t} \in \mathbb{R} \text { or } \mathbb{C} .
$$

Differing from (3), the resulted Fourier coefficient by GA is represented with the denotation of " $\langle\cdot\rangle_{k}$ ", which is a instantaneous variable including the small-signal dynamics, i.e, $\langle x\rangle_{k}(t)=X_{k}+\Delta x_{k}$. Thus, it is also named as dynamic phasor $(D P)$. The SSA is a special case of GA with $k=0$. Hence, the GA enables to characterize dynamics beyond $\omega_{s} / 2$, which is usually used to model ac system dynamics with multiple harmonics in the steady-state trajectory. The $k$-th DP, $\langle x\rangle_{k}(t)$, characterizes the system dynamics within the frequency range $\left(k \omega_{s}-\omega_{s} / 2, k \omega_{s}+\omega_{s} / 2\right)$. The GA realizes a time-invariant representation of the original ac system, which is the basic transformation for the DP modeling. Since GA is essentially an inner product operation [41], it is a linear transformation.

\section{2) DQ Transformation}

The purpose of using the $d q$ (Park) transformation is to represent three-phase ac systems in the synchronous reference frame, which converts time-periodic variables into timeinvariant variables in three-phase balanced conditions. Given a three-phase signal denoted in the stationary reference frame by $\left[x_{\alpha}, x_{\beta}, x_{z}\right]^{\mathrm{T}}$, its $d q z$ transformation is defined as

$$
\left[\begin{array}{l}
x_{d} \\
x_{q} \\
x_{z}
\end{array}\right]=\left[\begin{array}{ccc}
\cos \theta & \sin \theta & 0 \\
-\sin \theta & \cos \theta & 0 \\
0 & 0 & 1
\end{array}\right]\left[\begin{array}{l}
x_{\alpha} \\
x_{\beta} \\
x_{z}
\end{array}\right],
$$

whose inverse exists to transform the signal back to the stationary reference frame. As the zero-sequence component is a decoupled variable from the $d q$ variables, it is neglected in the following derivation for simplicity. If needed, the zerosequence dynamics can be modeled independently.

Similar to the GA, the $d q$ transformation also realizes a time-invariant representation of the ac system, yet its principle is different from the Fourier series expansion shown in (7), since it merely applies a phase rotation related to $\theta$. And the resulted time-invariant presentation is only valid when the three-phase system is balanced.

The phase rotation $\theta$ in the $d q$ transformation is frequencyand time-dependent. When it is considered in the small-signal modeling, it can be defined in two ways, in respect to the frames of reference [8]:

a) $\theta=\omega_{s} t+\varphi_{s}$, where $\omega_{s}$ is the system steady-state frequency and $\varphi_{s}$ is the initial phase. The initial phase is usually considered, such that the derived $d q$-frame model is aligned with the $d q$ frame that is used for the converter control implementation. With this $\theta$, the ac signal is transformed into a $d q$ reference frame that is named as the system dq frame.

b) $\theta=\omega_{s} t+\varphi_{s}+\Delta \theta=\left(\omega_{s}+\Delta \omega\right) t+\varphi_{s}$, where a phase variation $\Delta \theta$ is considered additionally, which results from a frequency variation $\Delta \omega$. This rotation is typically used to model converter control systems, since the control is implemented in its own $d q$ frame, where the phase dynamic originates from the synchronization control unit. Thus, the reference frame with this transformation is named as the local $d q$ frame (or control $d q$ frame as called in [5]).

It is important to note that the transformation into local $d q$ frame is essential to characterize the phase dynamics brought by the synchronization control of converters [5]. However, the derived model can be represented in either system dq frame or local $d q$ frame, and the system $d q$ frame is recommended. See Part C-2) for more elaborations. For the GDQ transformation introduced in Part B-4), only the representation in system $d q$ frame will be discussed.

\section{3) Complex Transformation and Its Extension}

The stationary or synchronous reference frame variables can be represented by using real-space vectors or complex-space vectors [7], [42], [43]. In time domain, their transformation can be realized by a complex transformation [42] matrix given by

$$
\left[\begin{array}{c}
\mathbf{x}_{\alpha \beta} \\
\mathbf{x}_{\alpha \beta}^{*}
\end{array}\right]=\left[\begin{array}{cc}
1 & j \\
1 & -j
\end{array}\right]\left[\begin{array}{l}
x_{\alpha} \\
x_{\beta}
\end{array}\right],
$$

which also applies to $d q$-frame variables. It is noted that the second complex variable in the left vector is the conjugate of the first one, yet it is in theory not negligible, since it is linearly independent of the first one. The complex-vector representation can deal with frequency-related transformations using exponential functions, which benefits in modeling the system frequency-coupling dynamics by complex transfer functions [7], [11], [12]. Fig. 1 shows the time-domain mathematical relationship of the $d q$ transformation in real and complex spaces [30], [42], [43]. For simplicity, the four frames for the time-domain ac variable representation are called as real $\alpha \beta$ frame, real $d q$ frame, complex $\alpha \beta$ frame, and complex $d q$ frame in the rest of the paper. It is seen that the $d q$ transformation can be denoted in a more compact form using $e^{-j \theta}$ and $e^{j \theta}$ as diagonal elements in the transformation matrix, indicating that the $d q$ transformation merely results in a frequency shift in the frequency domain. 


\section{IEEE REGULAR PAPER}

The complex transformation can be also extended based on the Fourier series expansion. When the ac variables are represented by DPs of the $\alpha \beta$-frame variables, the complex transformation needs to be applied to each order of DPs, i.e., $\left\langle\mathbf{x}_{\alpha \beta}\right\rangle_{+k}=\left\langle x_{\alpha}\right\rangle_{+k}+j\left\langle x_{\beta}\right\rangle_{+k}$ and $\left\langle\mathbf{x}_{\alpha \beta}^{*}\right\rangle_{+k}=\left\langle x_{\alpha}\right\rangle_{+k}-j\left\langle x_{\beta}\right\rangle_{+k}$, yielding a higher-order transformation matrix as shown in Fig. 2. It is noted that the inputs and outputs of this transformation are both complex-valued, thus the transformation is named as extended complex transformation for distinction. It also applies to DPs or Fourier coefficients derived from $d q$-frame variables.

\section{4) GDQ Transformation with SSA}

The $d q$ transformation can be generalized in unbalanced or harmonically distorted conditions as the generalized $d q$ (GDQ) transformation [23] given by

$$
\left[\begin{array}{c}
x_{d k} \\
x_{q k} \\
\vdots \\
x_{d-k} \\
x_{q-k}
\end{array}\right]=\left[\begin{array}{ccccc}
\cos \theta_{k} & \sin \theta_{k} & & & \\
-\sin \theta_{k} & \cos \theta_{k} & & & \\
& & \ddots & & \\
& & & \cos \theta_{-k} & \sin \theta_{-k} \\
& & & -\sin \theta_{-k} & \cos \theta_{-k}
\end{array}\right]\left[\begin{array}{c}
x_{\alpha} \\
x_{\beta}
\end{array}\right],
$$

where $\theta_{k}=k \omega_{s} t+\varphi_{k}$ and $\theta_{-k}=-k \omega_{s} t+\varphi_{-k}$ are the phases of the positive- and negative $k$-th harmonic components, and their initial phases can be different. It is seen that the GDQ transformation maps the original signal into multiple $d q$ frames. This also allows characterizing dynamics of multiple harmonics in the steady-state trajectory with time-invariant variables.

The GDQ transformation can be similarly represented in the complex space by exponential functions according to Fig. 1, i.e.,

$$
\left[\begin{array}{c}
\mathbf{x}_{d q k} \\
\mathbf{x}_{d q k}^{*} \\
\vdots \\
\mathbf{x}_{d q-k} \\
\mathbf{x}_{d q-k}^{*}
\end{array}\right]=\left[\begin{array}{cc}
e^{-j \theta_{k}} & 0 \\
0 & e^{j \theta_{k}} \\
\vdots & \vdots \\
e^{-j \theta_{-k}} & 0 \\
0 & e^{j \theta_{-k}}
\end{array}\right]\left[\begin{array}{c}
\mathbf{x}_{\alpha \beta} \\
\mathbf{x}_{\alpha \beta}^{*}
\end{array}\right],
$$

It is indicated that the GDQ transformation itself only results in frequency shifts of the original ac signal by the phase rotations. If the steady-state trajectory of the ac signal has multiple harmonics in the $\alpha \beta$ frame, the resulted $d q$-frame variables are still time-periodic. The harmonic components in the multiple $d q$-frame variables are redundant. In order to obtain the time-invariant representation, the SSA operator needs to be further applied, i.e.,

$$
\left[x_{d k}, x_{q k}\right]^{\mathrm{T}} \stackrel{\mathrm{SSA}}{\longrightarrow}\left[\bar{x}_{d k}, \bar{x}_{q k}\right]^{\mathrm{T}},
$$

where the averaging time window is chosen as the fundamental period of the resulted $d q$-frame variables. In this way, only the dc components will be extracted. By applying the SSA, the reversibility of the GDQ transformation can also be ensured. The original ac signal can thus be represented as

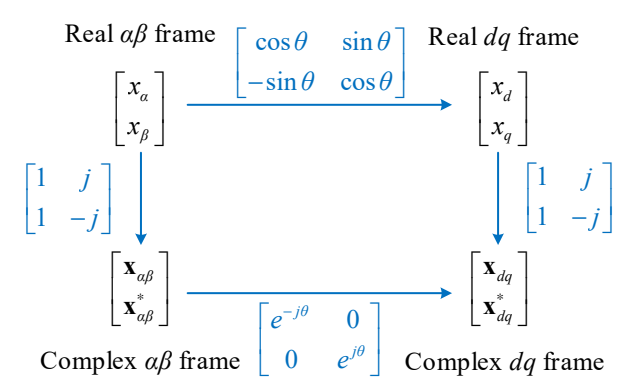

Fig. 1 Time-domain mathematical relationships in $\alpha \beta$ and $d q$ frame [30], [42].

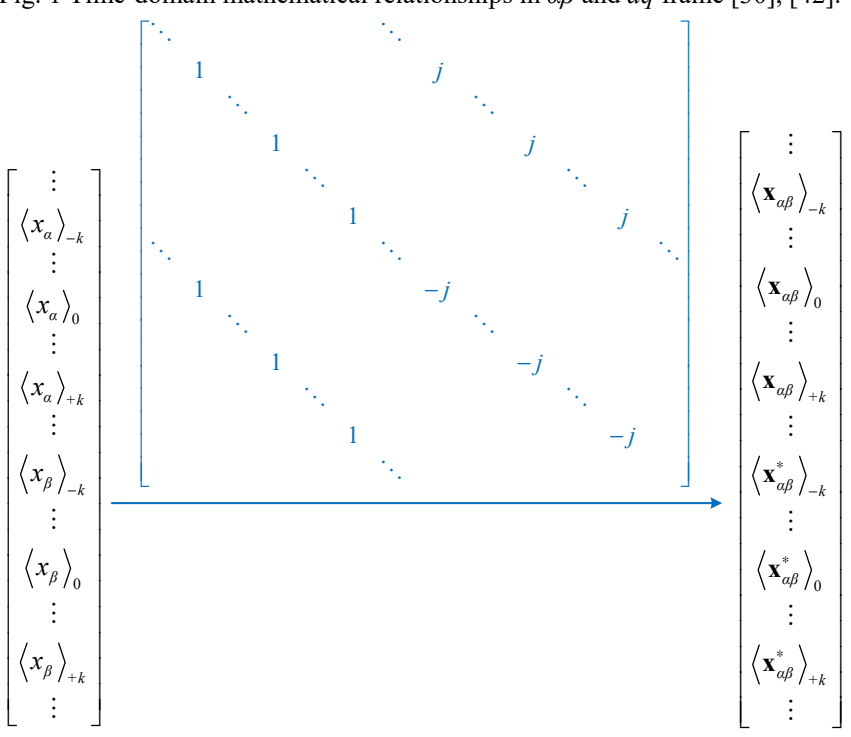

Fig. 2 Extended complex transformation based on Fourier series expansion.

$$
\left[\begin{array}{l}
x_{\alpha} \\
x_{\beta}
\end{array}\right]=\sum_{k}\left[\begin{array}{cc}
\cos \theta_{k} & -\sin \theta_{k} \\
\sin \theta_{k} & \cos \theta_{k}
\end{array}\right]\left[\begin{array}{l}
\bar{x}_{d k} \\
\bar{x}_{q k}
\end{array}\right] .
$$

It can be seen that the GDQ transformation with SSA also realizes a linear representation of the original ac signal with time-invariant $d q$-frame variables, where the orthogonal basis is the set of triangular function matrices for all the $k$ shown in (13). Here, the orthogonal basis is also determined by the system steady-state frequencies $k \omega_{\mathrm{s}}$, since the initial phase is merely a scalar, which does not change the frequency. The GDQ transformation with SSA is essential for the GDQ modeling.

\section{Linearization}

\section{1) Taylor Series}

A nonlinear dynamic system can be linearized around the given points $x_{0}$ and $u_{0}$ based on the Taylor series expansion [27], i.e.,

$$
\begin{aligned}
\dot{x}=f(x, u)= & f\left(x_{0}, u_{0}\right)+\left.\frac{\partial f}{\partial x}\right|_{\substack{x=x_{0} \\
u=u_{0}}}\left(x-x_{0}\right) \\
& +\left.\frac{\partial f}{\partial u}\right|_{\substack{x=x_{0} \\
u=u_{0}}}\left(u-u_{0}\right)+R\left(x-x_{0}, u-u_{0}\right)
\end{aligned} .
$$

provided that those partial derivatives exist. Eq. (14) applies to both real-valued or complex-valued equations. By neglecting 


\section{IEEE REGULAR PAPER}

the higher-order terms $R\left(x-x_{0}, u-u_{0}\right)$, a small-signal model can be obtained as

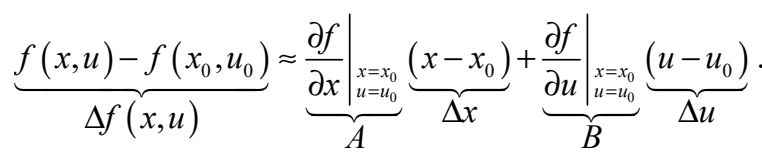

This linearization applies to both time-invariant and timevarying dynamic systems. For the time-invariant systems, which can be obtained based on GA or DQ/GDQ transformations, $x_{0}$ and $u_{0}$ are equilibrium points represented by $X_{0}$ and $U_{0}$, thus the resulted LTI model has constant $A$ and $B$.

For time-varying systems where $x_{0}$ and $u_{0}$ are time-varying trajectories represented by $x_{0}(t)$ and $u_{0}(t)$, a linear time-varying system with time-varying coefficients can be obtained, whose state-space form is given by

$$
\begin{aligned}
& \dot{x}(t)=A(t) x(t)+B(t) u(t) \\
& y(t)=C(t) x(t)+D(t) u(t)
\end{aligned}
$$

where the " $\Delta$ " before signals is neglected for simplicity. Since the ac system is $T$-periodic in steady state, the linearization results in an LTP system. All the signals and coefficients in (15) become $T$-periodic. Therefore, within $t \in[0, T)$, the signals and the coefficients can be represented by the form of

$$
\begin{gathered}
x(t)=\sum_{k} x_{k} e^{j k \omega_{s} t}, \text { similar for } u(t), y(t), \\
A(t)=\sum_{k} A_{k} e^{j k \omega_{s} t}, \text { similar for } B(t), C(t), D(t),
\end{gathered}
$$

where $\omega_{s}=2 \pi / T$. The linearization of time-periodic systems and the Fourier series representation in (17) and (18) are the bases of the HSS modeling.

\section{2) $D Q / G D Q$ Model Representation}

For the $d q / \mathrm{GDQ}$-frame modeling of ac converter systems, the dynamic of phase angle $\Delta \theta$ has to be considered in the linearization, in order to characterize synchronization control dynamics. The resulted small-signal model can be represented in either the system $d q$ frame or the local $d q$ frame, yet the model input and output variables are different.

Fig. 3 shows the the input-output dynamic modeling for a converter system $d q$-frame modeling. The nonlinear dynamics are shown at the top of Fig. 3, where the phase angle $\theta$ obtained from the converter control is used for the $d q$ transformation and the inverse $d q$ transformation. In order to model the phase dynamic $\Delta \theta$, the $d q$ or the inverse $d q$ transformation is intentionally decomposed into two transformations in cascade, yielding two distinguished dq frames, i.e., the system $d q$ frame and the local $d q$ frame. Taking the $d q$ transformation applied to the input variable $(u)$ for example, the first $d q$ transformation (Step 1) is merely related to the steady-state phase $\omega_{s} t+\varphi_{s}$, which is thus a linear transformation. It rotates the $\alpha \beta$-frame variables into the system $d q$ frame. The second $d q$ transformation (Step 2) is nonlinear, since $\Delta \theta$ includes dynamics from converter control. Similarly, the inverse $d q$ transformation applied to the output variable $(y)$ can be represented in an inverse way by Step 4 and Step 5.

Since Step 2 and Step 4 are nonlinear, whose linearization can be done with Taylor series expansion by assuming that $\Delta \theta$ is sufficiently small, such that $e^{-j \Delta \theta} \approx 1-j \Delta \theta$ and $e^{j \Delta \theta} \approx 1+j \Delta \theta$. The resulted small-signal dynamic path is shown at the bottom of Fig. 3, where two $d q$-frame model representations are illustrated. The blue shaded area indicates the small-signal model in the system $d q$ frame, where the input and output dynamics are represented by the system dq-frame variables $\left(\Delta \mathbf{u}_{d q}^{s}\right.$ or $\Delta \mathbf{y}_{d q}^{s}$ ) [44], [45], while $\Delta \theta$ is merely a state variable of the system.
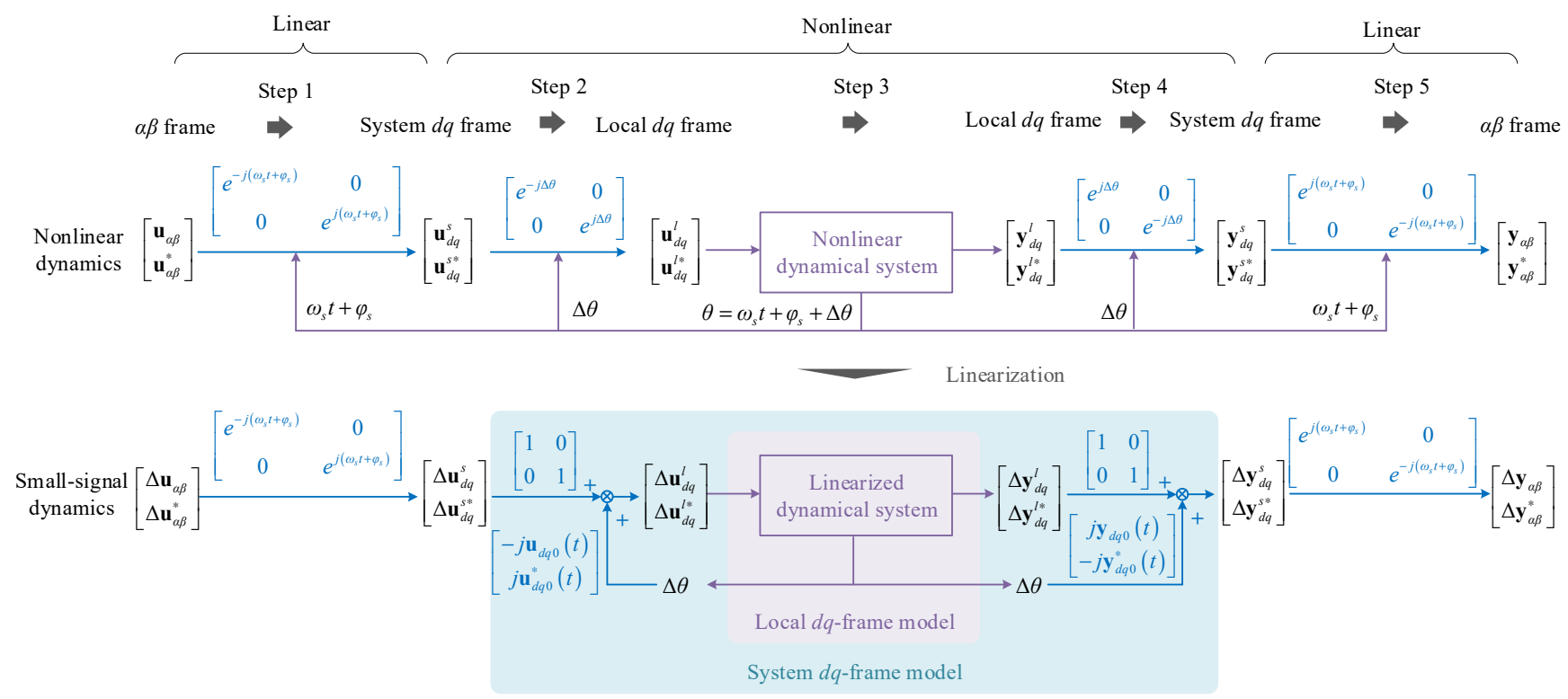

Fig. 3 Model representations in the system dq frame and the local dq frame. 


\section{IEEE REGULAR PAPER}

In contrast, if the model is represented in the local dq frame, as shown in the purple shaded area, the input and output dynamics need to be represented by both local dq-frame variables $\left(\Delta \mathbf{u}_{d q}^{l}\right.$ or $\left.\Delta \mathbf{y}_{d q}^{l}\right)$ and $\Delta \theta$. In such a case, $\Delta \theta$ becomes a necessary input or output variable [46].

The similar idea has already been reported in the modeling of resonant converters based on the phasor transformations [10], [47]. The conventional phasor transformation adopts the form of $e^{-j \omega_{s} t}$ [10], which is similar to the $d q$ transformation into the system $d q$ frame. As for the modified phasor transformation in [47], it considers a frequency dynamic in the transformation, i.e., $e^{-j\left(\omega_{s} t+\Delta \omega t\right)}$, which is similar to the $d q$ transformation into the local dq frame.

Compared with the local dq-frame representation, the system dq-frame representation has a simpler form for the input and output variables, which is thus more recommended for the $d q /$ GDQ model representation.

\section{Common Mathematical Bases}

While linearized modeling methods adopt different steadystate representations and transformations, they share common mathematical bases, which can be summarized as follows:

1) A $T$-periodic ac system can always be interpreted by the linear superposition of time-invariant systems, with an orthogonal basis determined by system steady-state frequencies $k \omega_{\mathrm{s}}$. Although the LTP model is directly denoted by $T$-periodic functions in the time-domain, it can be further transformed in the frequency domain by the HSS modeling that is based on the Fourier series, which shares the same mathematical basis as the DP modeling. The same orthogonal basis is used for the HSS modeling and DP modeling according to (7) and (17), which implies that the derived frequency-domain responses have the same input and output variables. The GDQ modeling adopts a different transformation for time-invariant representation of the system, which results in a different orthogonal basis given in (13).

2) Once the orthogonal basis is defined, the system input and output dynamics can be directly characterized with the time-invariant variables under this orthogonal basis, e.g., the Fourier coefficients in the HSS and DP modeling and the multiple $d q$-frame variables in the GDQ modeling under the system $d q$ frame. It is worth noting that the local dq-frame representation of the GDQ model does not agree with this principle, since in the HSS or DP model, $\Delta \theta$ is also regarded as a state variable, instead of a necessary input or output for ac variables (e.g., seeing (9k) and (9l) in [22]). The system small-signal model can be derived based on the harmonic balance on both sides of dynamical equations [48]: due to the linear independent nature of the orthogonal basis, the considered number of basis functions (harmonics) on both sides of the equation needs to be the same, and the total sum of the coefficients of each basis function (harmonic) on both sides of the equation should be equal.

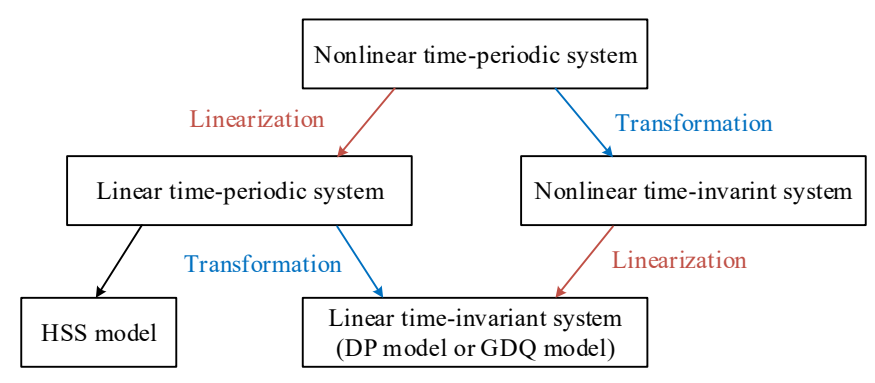

Fig. 4 Sequence of linearization and transformation in the three modeling methods.

\section{MATHEMATICAL RELATIONSHIPS}

This section compares principles of the HSS, the DP, and the GDQ modeling methods, and reveals their mathematical relationships explicitly, where the GDQ model is represented in the system dq frame.

\section{A. Sequence of Linearization and Transformation}

To characterize control dynamics of an ac converter system, the SSA operator is commonly used to filter out the converter switching dynamics, which leads to an averaged model that is nonlinear and time-periodic.

Fig. 4 provides an overview on the sequence of linearization and transformation in the three modeling methods. In the HSS modeling, the linearization is directly performed on the timeperiodic operating trajectories, resulting in an LTP dynamic system without further transformations. In contrast, the DP and the GDQ modeling methods apply transformations first to obtain a nonlinear time-invariant model, and then perform the linearization on time-invariant operating points, leading to LTI systems.

While the transformation and linearization are performed sequentially in the DP and GDQ modeling, they are exchangeable since the used transformations are linear, i.e., one can either do the linearization first around steady-state operating trajectories and then apply the transformation to get the LTI system, or apply the transformation first to obtain a time-invariant system and then linearize the system around the steady-state operating point.

Fig. 5 provides an overview of the principles of the HSS, the DP, and the GDQ modeling methods. Thanks to the interchangeability of transformation and linearization, the DP and GDQ modeling methods are elaborated directly based on an LTP model. Detailed modeling principles of the three methods are given in Parts $B-D$, respectively, and their mathematical relationships are derived explicitly in Part $E$.

\section{B. HSS Modeling}

The HSS modeling method directly derives the frequency response of an LTP system, which is achieved by introducing the exponential function $e^{s t}$ to time-periodic signals, namely the exponentially modulated periodic (EMP) signal [26], which is given by

$$
x(t)=e^{s t} \sum_{k} x_{k} e^{j k \omega_{s} t}
$$




\section{IEEE REGULAR PAPER}

where $s \in \mathbb{C}$. The same form also applies to input and output signals, i.e., $u(t)$ and $y(t)$.

Given an LTP system, if the input is an EMP signal, the output is still an EMP signal [26], [29]. Using EMP signals enables to derive the frequency-domain dynamic of an LTP system, since the frequency-dependent variable " $s$ " is introduced to represent dynamics. Based on the harmonic balance between input and output EMP signals, the frequencydomain dynamics of an LTP system can be analogous to an LTI transfer function between different Fourier series coefficients.

Fig. 6 illustrates the three steps of deriving the frequencydomain input-output dynamics of an LTP system:

1) The EMP signals are introduced to all the time-periodic variables, including the input and output variables, as well as the state variables of the LTP system.

2) The Fourier series expansion is then performed on both sides of the time-periodic dynamical equation. Due to the orthogonality of basis functions, the original dynamical equation can be decomposed into multiple equations, where each is related to one basis function, i.e. $e^{j k \omega_{s} t}$. The number of $k$ can be determined based on the model truncation considering major harmonics [26].

3) For the dynamical equation of each basis function, the time-dependent exponential functions can be canceled. Then, only the Fourier coefficients and the " $s$ " variable are retained, such that an LTI transfer function $(\boldsymbol{G}(s))$ matrix is formulated to represent the input-output dynamics of the LTP system, which is named as HTF.

Applying the abovementioned steps to the LTP state-space model, the HSS model [26] can be obtained as

$$
\begin{aligned}
& s x=(\mathcal{A}-\mathcal{N}) x+\mathcal{B} u \\
& y=C x+\mathcal{D} u
\end{aligned}
$$

where the signals are given by the Fourier coefficients $x=[\cdots$, $\left.x_{-k}, \cdots, x_{0}, \cdot \cdot x_{k}, \cdots\right]^{\mathrm{T}}$ according to (17), and similar for $\boldsymbol{u}$ and $\boldsymbol{y}$. The coefficient matrices are represented by the Toeplitz matrices, which are given by their Fourier coefficients according to (18), e.g.,

$$
\mathcal{A}=\left[\begin{array}{ccccc}
\ddots & \vdots & \vdots & \vdots & \cdot \\
\cdots & A_{0} & A_{-1} & A_{-2} & \cdots \\
\cdots & A_{1} & A_{0} & A_{-1} & \cdots \\
\cdots & A_{2} & A_{1} & A_{0} & \cdots \\
\cdot & \vdots & \vdots & \vdots & \ddots
\end{array}\right], \text { similar for } \mathcal{B}, C, \mathcal{D} .
$$

$\mathcal{N}$ is a diagonal matrix given by

$$
\mathcal{N}=\operatorname{diag}\left\{\begin{array}{lllllll}
\cdots & -j k \omega_{s} & \cdots & 0 & \cdots & j k \omega_{s} & \cdots
\end{array}\right\} .
$$

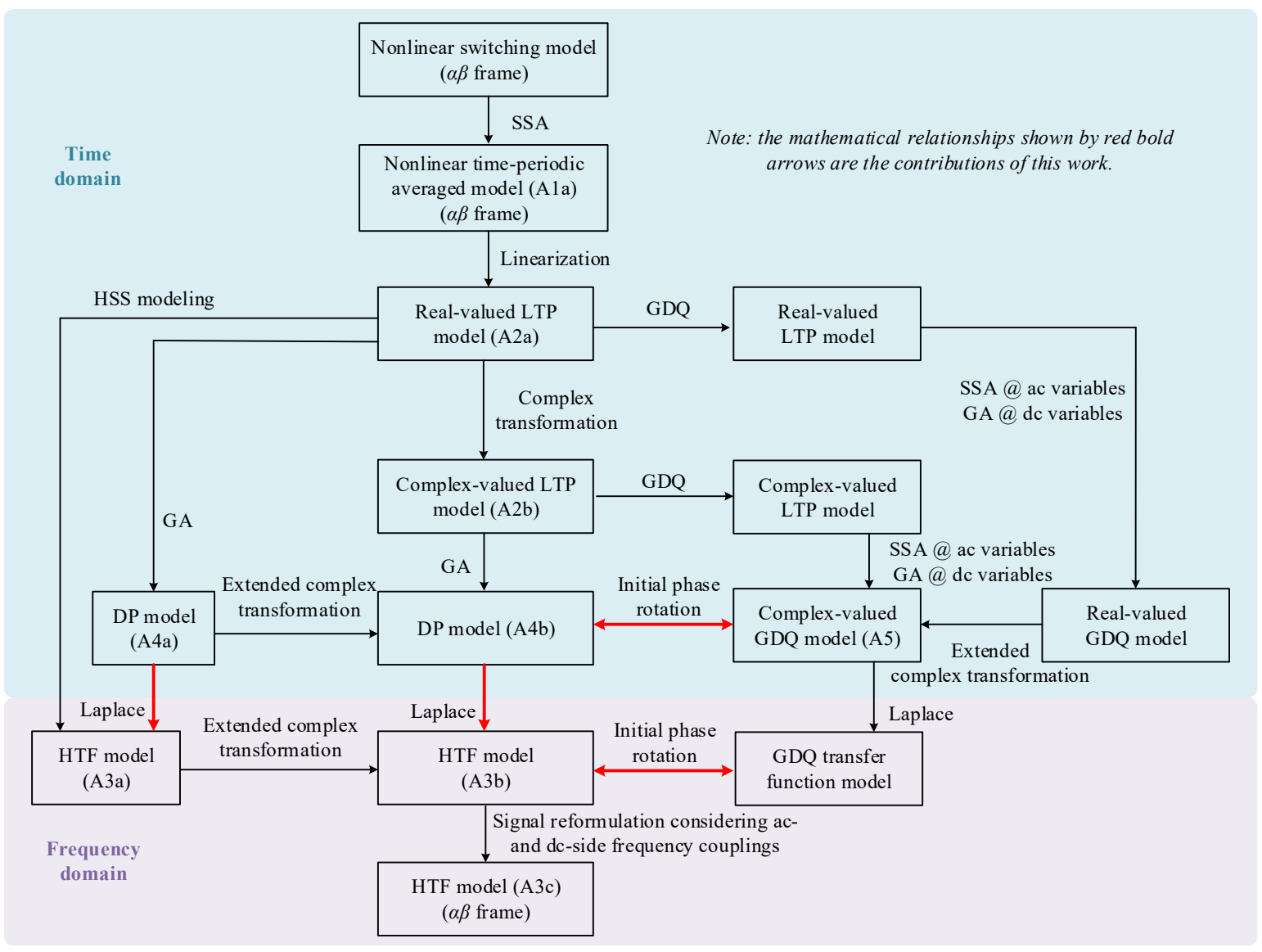

Fig. 5 Mathematical relationships between different modeling methods for ac power electronic systems. 


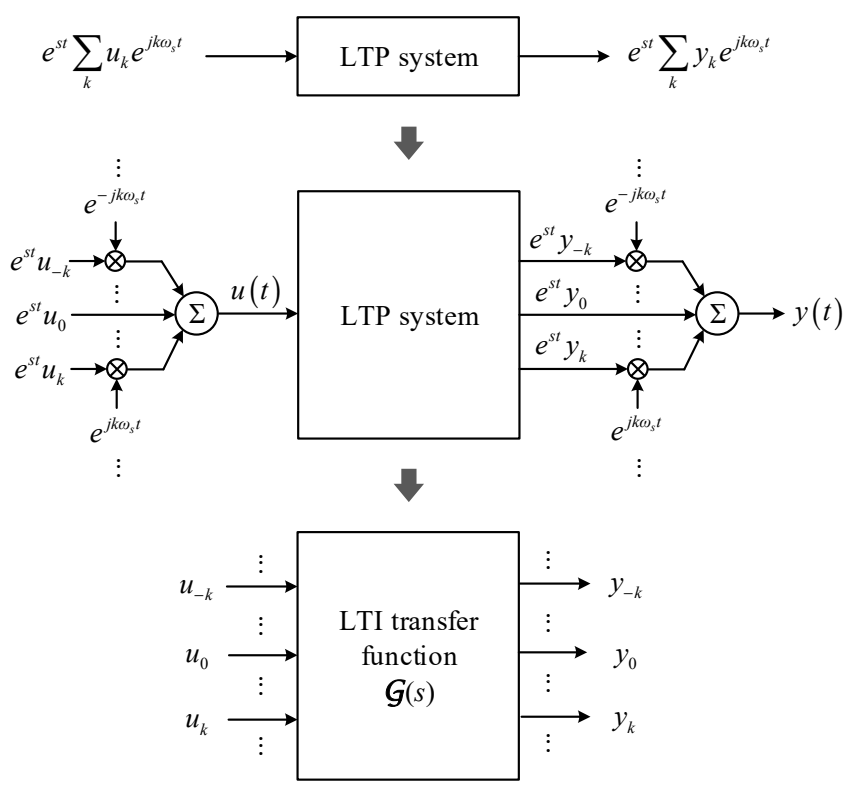

Fig. 6 The frequency response of an LTP system represented by an LTI transfer function.

It is clear that the HSS model is a frequency-domain model represented in the state-space form, based on which the HTF from the input $u$ to the output $y$ can be derived as

$$
\boldsymbol{G}(s)=C[s \mathbf{I}-(\mathcal{A}-\mathcal{N})]^{-1} \mathcal{B}+\mathcal{D},
$$

where $\mathbf{I}$ denotes the identity matrix.

The HSS modeling is usually derived based on real-valued LTP models, such as in real $\alpha \beta$ frame or real $d q$ frame, where the HTFs of the coefficient matrices are Hermitian according to (21). The HTF model can also be transformed through an extended complex transformation according to Fig. 2, to characterize the input-output dynamics of the complex-valued LTP model [16]-[18].

\section{DP Modeling}

By applying GA operators to the LTP system in (16), the DP model can be derived. For a generic ac system with multiple harmonics, multiple DPs are needed. First, the differential equations of the $k$-th DP can be derived based on the principle of harmonic balance, which are given as

$$
\begin{aligned}
& \frac{\mathrm{d}\langle x\rangle_{k}}{\mathrm{~d} t}=-j k \omega_{s}\langle x\rangle_{k}+\sum_{m} A_{k-m}\langle x\rangle_{m}+\sum_{m} B_{k-m}\langle u\rangle_{m} . \\
& \langle y\rangle_{k}=\sum_{m} C_{k-m}\langle x\rangle_{m}+\sum_{m} D_{k-m}\langle u\rangle_{m}
\end{aligned}
$$

Then, incorporating the differential equations of different DPs, the DP model of the entire system can be represented in the state-space form [20] as

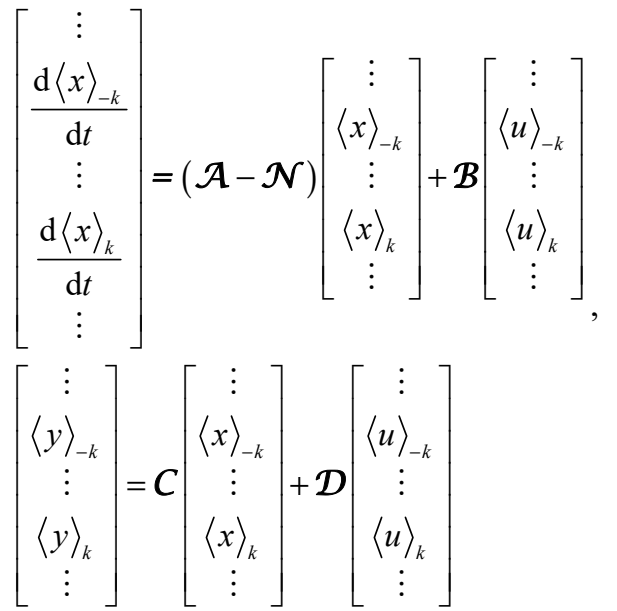

where the coefficient matrices are found the same as those in (20).

The DP modeling can be applied to both real-valued models (e.g., real $\alpha \beta$ frame or real $d q$ frame) and complex-valued models (e.g., complex $\alpha \beta$ frame). This work merely discusses the DP models derived from the $\alpha \beta$-frame LTP models, as shown in Fig. 5. Some examples of DP models derived from the $d q$-frame LTP models can be found in [19], [22], which mainly differ in a different selection of DP orders.

\section{GDQ Modeling}

The GDQ model is derived from the $\alpha \beta$-frame model by applying the GDQ transformation with SSA. However, the differential equations of a converter system are composed of both ac and dc variables, such as $x_{\mathrm{z}}$ and $x_{\mathrm{dc}}$ shown in (26).

$$
\left[\begin{array}{c}
\frac{\mathrm{d} x_{\alpha}}{\mathrm{d} t} \\
\frac{\mathrm{d} x_{\beta}}{\mathrm{d} t} \\
\frac{\mathrm{d} x_{\mathrm{z}}}{\mathrm{d} t} \\
\frac{\mathrm{d} x_{\mathrm{dc}}}{\mathrm{d} t}
\end{array}\right]=\mathbf{A}(t)\left[\begin{array}{c}
x_{\alpha} \\
x_{\beta} \\
x_{z} \\
x_{\mathrm{dc}}
\end{array}\right]+\mathbf{B}(t)\left[\begin{array}{c}
u_{\alpha} \\
u_{\beta} \\
u_{z} \\
u_{\mathrm{dc}}
\end{array}\right] .
$$

Although the ac ( $\alpha \beta$-frame) variables can be represented by time-invariant representations in multiple $\mathrm{dq}$ frames, the $\mathrm{dc}$ variables can still be time-periodic, whose harmonics also need to be represented with time-invariant forms to ensure the harmonic balance of the entire system [23]. That is to say, to establish an accurate GDQ model, one has to apply:

1) GDQ transformation with SSA to ac variables;

2) GA to dc variables.

The above procedures applied to (26) yield the state-space GDQ model represented by 


$$
\left[\begin{array}{c}
\vdots \\
\frac{\mathrm{d} \bar{x}_{d k}}{\mathrm{~d} t} \\
\frac{\mathrm{d} \bar{x}_{q k}}{\mathrm{~d} t} \\
\vdots \\
\mathrm{d}\left\langle x_{z}\right\rangle_{k} \\
\mathrm{~d} t \\
\vdots \\
\mathrm{d}\left\langle x_{\mathrm{dc}}\right\rangle_{k} \\
\mathrm{~d} t \\
\vdots
\end{array}\right]=\left[\begin{array}{c}
\vdots \\
k \omega_{s} \bar{x}_{q k} \\
-k \omega_{s} \bar{x}_{d k} \\
\vdots \\
-j k \omega_{s}\left\langle x_{z}\right\rangle_{k} \\
\vdots \\
-j k \omega_{s}\left\langle x_{\mathrm{dc}}\right\rangle_{k} \\
\vdots
\end{array}\right]+\mathbf{A}_{\mathrm{GDQ}}\left[\begin{array}{c}
\vdots \\
\bar{x}_{d k} \\
\bar{x}_{q k} \\
\vdots \\
\left\langle x_{z}\right\rangle_{k} \\
\vdots \\
\left\langle x_{\mathrm{dc}}\right\rangle_{k} \\
\vdots
\end{array}\right]+\mathbf{B}_{\mathrm{GDQ}}\left[\begin{array}{c}
\vdots \\
\bar{u}_{d k} \\
\bar{u}_{q k} \\
\vdots \\
\left\langle u_{z}\right\rangle_{k} \\
\vdots \\
\left\langle u_{\mathrm{dc}}\right\rangle_{k} \\
\vdots \\
\end{array}\right]
$$

where $\mathbf{A}_{\mathrm{GDQ}}$ and $\mathbf{B}_{\mathrm{GDQ}}$ are the resulted coefficient matrices based on the harmonic balance. It is seen that the cross couplings between $d$ - and $q$-axis variables are resulted from the differential operation.

The GDQ model of (27) is represented in multiple real $d q$ frames, thus is named as real-valued GDQ model in Fig. 5. Alternatively, it can be transformed into multiple complex $d q$ frames through an extended complex transformation as the complex-valued GDQ model given by

$$
\left[\begin{array}{c}
\vdots \\
\frac{\mathrm{d} \overline{\mathbf{x}}_{d q k}}{\mathrm{~d} t} \\
\frac{\mathrm{d} \overline{\mathbf{x}}_{d q k}^{*}}{\mathrm{~d} t} \\
\vdots \\
\frac{\mathrm{d}\left\langle x_{z}\right\rangle_{k}}{\mathrm{~d} t} \\
\vdots \\
\frac{\mathrm{d}\left\langle x_{\mathrm{dc}}\right\rangle_{k}}{\mathrm{~d} t} \\
\vdots
\end{array}\right]=\left[\begin{array}{c}
\vdots \\
-j k \omega_{s} \overline{\mathbf{x}}_{d q k} \\
j k \omega_{s} \overline{\mathbf{x}}_{d q k}^{*} \\
\vdots \\
-j k \omega_{s}\left\langle x_{z}\right\rangle_{k} \\
\vdots \\
-j k \omega_{s}\left\langle x_{\mathrm{dc}}\right\rangle_{k} \\
\vdots
\end{array}\right]+\mathbf{A}_{\mathrm{GDQ}}^{\prime}\left[\begin{array}{c}
\vdots \\
\overline{\mathbf{x}}_{d q k} \\
\overline{\mathbf{x}}_{d q k}^{*} \\
\vdots \\
\left\langle x_{z}\right\rangle_{k} \\
\vdots \\
\left\langle x_{\mathrm{dc}}\right\rangle_{k} \\
\vdots
\end{array}\right]+\mathbf{B}_{\mathrm{GDQ}}^{\prime}\left[\begin{array}{c}
\vdots \\
\overline{\mathbf{u}}_{d q k} \\
\overline{\mathbf{u}}_{d q k}^{*} \\
\vdots \\
\left\langle u_{z}\right\rangle_{k} \\
\vdots \\
\left\langle u_{\mathrm{dc}}\right\rangle_{k} \\
\vdots
\end{array}\right],
$$

where the coefficient matrices $\mathbf{A}_{\mathrm{GDQ}}$ and $\mathbf{B}_{\mathrm{GDQ}}$ differ from $\mathbf{A}_{\mathrm{GDQ}}$ and $\mathbf{B}_{\mathrm{GDQ}}$ due to the extended complex transformation.

\section{E. Mathematical Relationships}

The mathematical relationships of the three modeling methods shown by red bold arrows in Fig. 5 are further elaborated.

\section{1) Relationship Between HSS and DP models}

By comparing (20) and (25), it can be found that the HSS model is a merely a frequency-domain model, while the DP model is a time-domain model. It is clear that the DP model can be further derived in the frequency domain by applying the Laplace transformation to (25), which yields the HSS model in (20).

The model representations in (20) and (25) are derived based on real-valued LTP models. The HTF and DP models can also be derived based on complex-valued LTP models
[16], [17], [19], [22], where the above relationship of Laplace transformation also applies.

\section{2) Relationship Between DP and GDQ models}

By comparing (28) and (24), it can be seen that the crosscoupling terms of the GDQ model have the same form as those in the DP model, since the cross coupling coefficient of $\overline{\mathbf{x}}_{d q k}$ agrees with the cross coupling coefficient of $\langle x\rangle_{k}$. This is because the averaged complex-valued $d q$-frame variables can be represented as

$$
\begin{aligned}
& \overline{\mathbf{x}}_{d q k}=\frac{1}{T} \int_{t-T}^{t} \mathbf{x}_{\alpha \beta} e^{-j\left(k \omega_{s} t+\varphi_{k}\right)} d \tau=e^{-j \varphi_{k}}\left\langle\mathbf{x}_{\alpha \beta}\right\rangle_{k} . \\
& \overline{\mathbf{x}}_{d q k}^{*}=\frac{1}{T} \int_{t-T}^{t} \mathbf{x}_{\alpha \beta}^{*} e^{j\left(k \omega_{s} t+\varphi_{k}\right)} d \tau=e^{j \varphi_{k}}\left\langle\mathbf{x}_{\alpha \beta}^{*}\right\rangle_{k}
\end{aligned} .
$$

It is clear that the GDQ model can be transformed from the DP model considering the initial phase rotation.

\section{3) Relationship Between HSS and GDQ models}

The GDQ model also results in an LTI state-space model in the time domain, which can be further transformed in the frequency domain by the Laplace transformation. The resulted GDQ frequency-domain model can also be transformed into the HSS model by the initial phase rotation.

\section{4) HTF Model Representation in Complex $\alpha \beta$ Frame}

Since the HTF and DP models can be transformed from the GDQ model through an initial phase rotation, it is implied that the HTF and DP models are essentially "multiple $d q$-frame" models, whose frequency-domain transfer function matrices are LTI. This can be easily understood, since the inputs and outputs of the HTF or DP model are all Fourier coefficients, which are exactly the time-invariant variables in multiple rotating reference frames.

However, the inputs and outputs can still be represented in the stationary frame through the signal reformulation. The acand dc-side frequency-coupling dynamics of the converter systems can be captured by a three-port model, where all the ac variables are represented by the complex-valued vector of $\left[\mathbf{x}_{\alpha \beta}, e^{j 2 \theta_{s}} \mathbf{x}_{\alpha \beta}^{*}\right]^{\mathrm{T}}$, and all the dc variables can be denoted by the complex-valued scalar of $e^{j \theta_{s}} x_{\mathrm{dc}}$ [12]. For the variable $\mathbf{x}_{\alpha \beta}$, its Fourier coefficients $\left(\mathbf{x}_{\alpha \beta k}, k \in \mathbb{Z}\right)$ and the corresponding frequency responses can be represented by

$$
\left[\begin{array}{c}
\vdots \\
\mathbf{x}_{\alpha \beta-k} \\
\vdots \\
\mathbf{x}_{\alpha \beta 0} \\
\vdots \\
\mathbf{x}_{\alpha \beta+k} \\
\vdots
\end{array}\right]=\left[\begin{array}{c}
\frac{\vdots}{e^{j k \omega_{s} t} \mathbf{x}_{\alpha \beta}(t)} \\
\vdots \\
\frac{\mathbf{x}_{\alpha \beta}(t)}{\vdots} \\
\frac{\vdots}{e^{-j k \omega_{s} t} \mathbf{x}_{\alpha \beta}(t)} \\
\vdots
\end{array}\right] \leftrightarrow\left[\begin{array}{c}
\vdots \\
\mathbf{X}_{\alpha \beta-k}(s) \\
\vdots \\
\mathbf{X}_{\alpha \beta 0}(s) \\
\vdots \\
\mathbf{X}_{\alpha \beta+k}(s) \\
\vdots \\
\uparrow \\
\text { multi-dq } \\
\text { stationary }
\end{array}\right]=\left[\begin{array}{c}
\vdots \\
\mathbf{X}_{\alpha \beta}\left(s-j k \omega_{s}\right) \\
\vdots \\
\mathbf{X}_{\alpha \beta}(s) \\
\vdots \\
\mathbf{X}_{\alpha \beta}\left(s+j k \omega_{s}\right) \\
\vdots \\
\uparrow
\end{array}\right]
$$

It can be seen that with the signal reformulation in the stationary frame, the frequency coupling relationships are 
characterized by $s \pm j k \omega_{\mathrm{s}}$. And this signal reformulation by $\mathbf{x}_{\alpha \beta}$ does not change the corresponding HTF. However, as for the variables $e^{j 2 \theta_{s}} \mathbf{x}_{\alpha \beta}^{*}$ and $e^{j \theta_{s}} x_{\mathrm{dc}}$, the exponential operators of $e^{j 2 \theta_{s}}$ and $e^{j \theta_{s}}$ are applied. Consequently, they not only lead to frequency couplings in the frequency responses, as represented by

$$
\begin{gathered}
{\left[\begin{array}{c}
\vdots \\
e^{j 2 \theta_{s}} \mathbf{x}_{\alpha \beta-k}^{*} \\
\vdots \\
e^{j 2 \theta_{s}} \mathbf{x}_{\alpha \beta 0}^{*} \\
\vdots \\
e^{j 2 \theta_{s}} \mathbf{x}_{\alpha \beta+k}^{*} \\
\vdots
\end{array}\right] \leftrightarrow\left[\begin{array}{c}
\vdots \\
\mathbf{X}_{\alpha \beta}^{*}\left(s-j k \omega_{s}-j 2 \omega_{s}\right) \\
\vdots \\
\mathbf{X}_{\alpha \beta}^{*}\left(s-j 2 \omega_{s}\right) \\
\vdots \\
e^{j \theta_{s}} x_{\mathrm{dc}-k} \\
\vdots \\
e^{j \theta_{s}} x_{\mathrm{dc} 0} \\
\vdots \\
e^{j \theta_{s}} x_{\mathrm{dc}+k}^{*}\left(s+j k \omega_{s}-j 2 \omega_{s}\right) \\
\vdots
\end{array}\right],\left[\begin{array}{c}
X_{\mathrm{dc}}\left(s-j k \omega_{s}-j \omega_{s}\right) \\
\vdots \\
X_{\mathrm{dc}}\left(s-j \omega_{s}\right) \\
\vdots \\
X_{\mathrm{dc}}\left(s+j k \omega_{s}-j \omega_{s}\right) \\
\vdots
\end{array}\right],}
\end{gathered}
$$

but also result in frequency shifts in the corresponding HTFs due to the chain rule [7].

The variable representations shown in (30)-(32) provides a uniform framework to modeling the ac- and dc-side frequency couplings of converter systems. It also benefits in a less order selection for HTFs, which will be illustrated more clearly on the case study models in the next section.

\section{CASE STUDY}

The mathematical relationships of different modeling methods are validated by a case study on a three-phase converter in unbalanced grids. The open-loop models based on the HSS modeling, DP modeling and GDQ modeling of the converter are derived first. The mathematical relationships between the LTP model, the DP model and the GDQ model are verified in time domain. Then, a unified frequency-domain model of the three methods considering the closed-loop dynamics is derived and validated by the frequency scan. All the validations are performed on the averaged model in MATLAB/Simulink.

\section{A. System Description}

Fig. 7 shows the three-phase converter under study with a single-line representation. The input voltage is three-phase unbalanced. The converter has the current control (CC), the phase-locked loop (PLL) and the dc-link voltage control (DVC). The CC is implemented in $\alpha \beta$ frame with proportional + resonant controllers. The PLL adopts a notch-filtered synchronous-reference-frame PLL, in order to filter out the negative-sequence voltage components for synchronization. The DVC adopts a proportional + integral controller. The circuit and control parameters are listed in Table I.

\section{B. Open-Loop Modeling and Validation}

The open-loop model of the converter system is modeled based on the averaged model first. All the derived models are given in Appendix. The differential equations of the ac- and dc-side circuits can be derived as (A1a), which is a nonlinear time-periodic model represented in the real $\alpha \beta$ frame. In addition, the complex $\alpha \beta$-frame model representation is given in (A1b).

\section{1) LTP Modeling}

Through the direct linearization around the steady-state trajectories, the LTP models are derived as (A2a) and (A2b), where the steady-state trajectories are determined by the $\alpha \beta$ frame variables and the dc-link voltage.

If the variable representations shown in (30)-(32) are used, the complex $\alpha \beta$-frame model can be derived as (A2c), where the steady-state trajectories are determined by the $d q$-frame variables and the dc-link voltage. The benefits of using this representation include: i) the $d q$-frame variables have the same harmonics with the dc-link voltage, which simplifies the HTF order selection, as introduced in the following HSS modeling part; ii) the model becomes an LTI model under balanced grid conditions, since all the steady-state trajectories become timeinvariant.

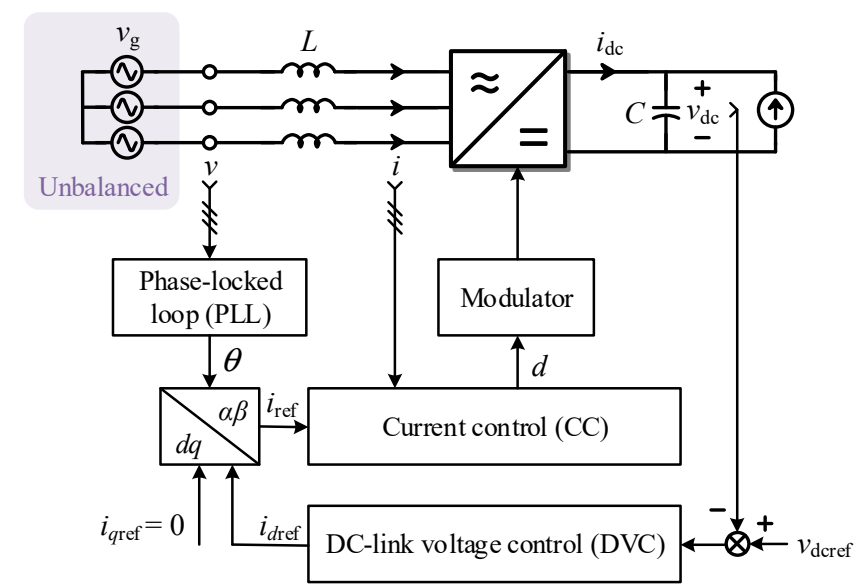

Fig. 7 Studied three-phase voltage-source converter under unbalanced grids.

TABLE I

VSC PARAMETERS

\begin{tabular}{c|c|c}
\hline \hline Parameters & Symbols & Values \\
\hline Grid voltage & $V_{p}$ & $1.0 \mathrm{p} . \mathrm{u} .(200 \mathrm{~V})$ \\
Fundamental frequency & $V_{n}$ & $0.5 \mathrm{p} . \mathrm{u}$. \\
Inverter L filter & $\omega_{s}$ & $2 \pi \cdot 50 \mathrm{rad} / \mathrm{s}$ \\
DC voltage & $L$ & $2 \mathrm{mH}$ \\
DC current & $V_{\mathrm{dc}}$ & $600 \mathrm{~V}$ \\
DC-side capacitance & $I_{\mathrm{dc}}$ & $3 \mathrm{~A}$ \\
$q$-axis current reference & $C$ & $0.45 \mathrm{mF}$ \\
Current PR Controller & $I_{q}$ & $0 \mathrm{~A}$ \\
& $K_{\mathrm{pi}}$ & $5 \Omega$ \\
PLL PI controller and notch filter & $K_{\mathrm{ri}}$ & $800 \Omega / \mathrm{s}$ \\
damping factor & $K_{\mathrm{ppll}}$ & $0.58 \mathrm{rad} /(\mathrm{s} \cdot \mathrm{V})$ \\
& $K_{\mathrm{ipll}}$ & $27.2 \mathrm{rad} /\left(\mathrm{s}^{2} \cdot \mathrm{V}\right)$ \\
DC-link voltage controller & $D_{p}$ & 0.707 \\
& $K_{\mathrm{pdvc}}$ & $0.5 \mathrm{~S}$ \\
\hline \hline
\end{tabular}




\section{2) HSS Modeling}

The frequency domain representation using HTFs can be further derived as (A3a)-(A3c), corresponding to the LTP models of (A2a)-(A2c), respectively. Although the model is not strictly represented in the HSS form, the derivation follows the same principle of the HSS modeling.

It can be found that in (A3a) and (A3b), the HTFs of steadystate trajectories are selected based on $\alpha \beta$-frame variables, which will lead to a higher-order selection for HTFs. Since the fundamental frequency of the $\alpha \beta$-frame variables is $\omega_{s}$, at least the orders of $0, \pm 1, \pm 2, \pm 3$ need to be considered under unbalanced grid conditions. As for (A3c), the HTFs of the steady-state trajectories are derived based on $d q$-frame variables, which results in a less-order selection for HTFs. Under unbalanced grid conditions, the fundamental frequency of the $d q$-frame variables is $2 \omega_{s}$, which is the same as $V_{\mathrm{dc}}(t)$. Thus, the major harmonic orders only include 0 and \pm 2 . Another feature coming with (A3c) is that the HTFs of $Z_{L}$ and $\mathcal{Y}_{C}$ are frequency shifted in the dynamical equations of $e^{j 2 \theta_{s}} \mathbf{v}_{\alpha \beta}^{*}$ and $e^{j \theta_{s}} v_{\mathrm{dc}}$, according to the chain rule brought by $e^{j 2 \theta_{s}}$ and $e^{j \theta_{s}}[7]$.

\section{3) DP Modeling}

The DP models can be derived as (A4a) and (A4b) based on (A2a) and (A2b), respectively. Applying the Laplace transformation yields the same frequency-domain models as (A3a) and (A3b).

\section{4) GDQ Modeling}

Considering the relationship between the GDQ model and the DP model, the complex-valued GDQ model is derived from (A4b) as (A5), where the major harmonic components $(+3,+1,-1)$ under an unbalanced grid condition are considered for a simplified model representation. It is found that, based on the defined GDQ transformation, an initialphase related rotation matrix $Q$ is involved in the model. Such a rotation also affects the differential equations of $v_{\mathrm{dc}}$, thus, the final dc-side voltage variables $\left(\mathbf{v}_{\mathrm{dc}}\right)$ in the GDQ model is not the original DPs $\left(\left\langle v_{\mathrm{dc}}\right\rangle\right)$ obtained by GA. (A5) can be further derived in the real space by the complex-to-real transformation if needed.

\section{5) Time-Domain Validation}

To validate the relationship between different models, the time-domain LTP model, the DP model, and the GDQ model are established in MATLAB/Simulink, to be compared with the averaged model. The DP model considers the same harmonic orders as the GDQ model. The equivalence of these models is validated by the time-domain waveforms of the $\alpha \beta$ frame currents and the dc-link voltage. Considering the mathematical relationship, the $\alpha \beta$-frame currents and dc-link voltage waveform of the DP model can be calculated by DPs by

$$
i_{\alpha}(t)=\operatorname{Re}\left[\left\langle\mathbf{i}_{\alpha \beta}\right\rangle_{+1} e^{j \omega_{s} t}+\left\langle\mathbf{i}_{\alpha \beta}\right\rangle_{-1} e^{-j \omega_{s} t}+\left\langle\mathbf{i}_{\alpha \beta}\right\rangle_{+3} e^{j 3 \omega_{s} t}\right]
$$

$$
\begin{gathered}
i_{\beta}(t)=\operatorname{Im}\left[\left\langle\mathbf{i}_{\alpha \beta}\right\rangle_{+1} e^{j \omega_{s} t}+\left\langle\mathbf{i}_{\alpha \beta}\right\rangle_{-1} e^{-j \omega_{s} t}+\left\langle\mathbf{i}_{\alpha \beta}\right\rangle_{+3} e^{j 3 \omega_{s} t}\right], \\
v_{\mathrm{dc}}(t)=\left\langle v_{\mathrm{dc}}\right\rangle_{0}+\left\langle v_{\mathrm{dc}}\right\rangle_{+2} e^{j 2 \omega_{s} t}+\left\langle v_{\mathrm{dc}}\right\rangle_{-2} e^{-j 2 \omega_{s} t} .
\end{gathered}
$$

The $\alpha \beta$-frame currents and dc-link voltage waveform of the GDQ model can be calculated by

$$
\begin{gathered}
i_{\alpha}(t)=\operatorname{Re}\left[\overline{\mathbf{i}}_{d q+1} e^{j \theta_{p}}+\overline{\mathbf{i}}_{d q-1} e^{j \theta_{n}}+\overline{\mathbf{i}}_{d q+3} e^{j\left(2 \theta_{p}-\theta_{n}\right)}\right], \\
i_{\alpha}(t)=\operatorname{Im}\left[\overline{\mathbf{i}}_{d q+1} e^{j \theta_{p}}+\overline{\mathbf{i}}_{d q-1} e^{j \theta_{n}}+\overline{\mathbf{i}}_{d q+3} e^{j\left(2 \theta_{p}-\theta_{n}\right)}\right], \\
v_{\mathrm{dc}}(t)=v_{\mathrm{dc} 0}+v_{\mathrm{dc}+2} e^{j\left(\theta_{p}-\theta_{n}\right)}+v_{\mathrm{dc}-2} e^{j\left(\theta_{n}-\theta_{p}\right)} .
\end{gathered}
$$

Then, the time-domain waveforms of the averaged (AVG) model, the LTP model, the DP model, and the GDQ model are compared in Fig. 8. At $0.1 \mathrm{~s}$, the negative-sequence voltage steps from 1 p.u. to 0.5 p.u.. It is found that all the models agree with each other, such that the mathematical relationship between the LTP model and the DP/GDQ model can be verified. The DP model has some time delay in the agreement, since the DPs are calculated by a moving averaging window.

To further verify the mathematical relationships between the DP model and the GDQ model, Fig. 9 compares different DPs and multiple $d q$-frame variables considering the initialphase rotation impacts. For easier plotting in the time domain, the real and imaginary parts of the complex-valued DPs and multiple $d q$-frame variables are calculated and compared. It can be seen that, after applying the initial phase rotation to the multiple $d q$-frame variables, the waveforms agree with the DPs.

\section{Closed-Loop Modeling and Validation}

As the mathematical relationships of different models have been validated on the open-loop model, it can be inferred that all these models yield a unified frequency-domain representation through proper transformations. In this part, a unified closed-loop HTF model considering the frequency couplings in the complex $\alpha \beta$ frame is derived, which is validated by the frequency scan.

For the closed-loop modeling, the modular modeling is considered. The general flow is given in Fig. 10. The converter system is partitioned into different subsystems. For nonlinear subsystems, their HTF models can be derived based on the linearized modeling. For linear subsystems, their HTF models can be simply derived by LTI transfer functions considering frequency shifts. Such a modular modeling method is readily applicable to converters with different control schemes and also to systems with multiple converters.

For the converter system in Fig. 7, the open-loop circuit model, the PLL and the DVC are three nonlinear subsystems, while the $\mathrm{CC}$ and the time delay are linear subsystems. The linearized modeling of the PLL and DVC subsystems can be done similarly as what have been done for the open-loop circuit modeling. To ensure an easy interconnection of different subsystems, the ac- and dc-side variables are uniformly defined in the complex $\alpha \beta$ frame considering their frequency-coupling relationships, as introduced in Section IIIE-4). The ac-side variables are defined by $\mathbf{v}_{\mathrm{ac}}$ and $\mathbf{i}_{\mathrm{ac}}$ shown in Fig. 11 and the dc-link voltage is defined by $e^{j \theta_{s}} v_{\mathrm{dc}}$. In such a 


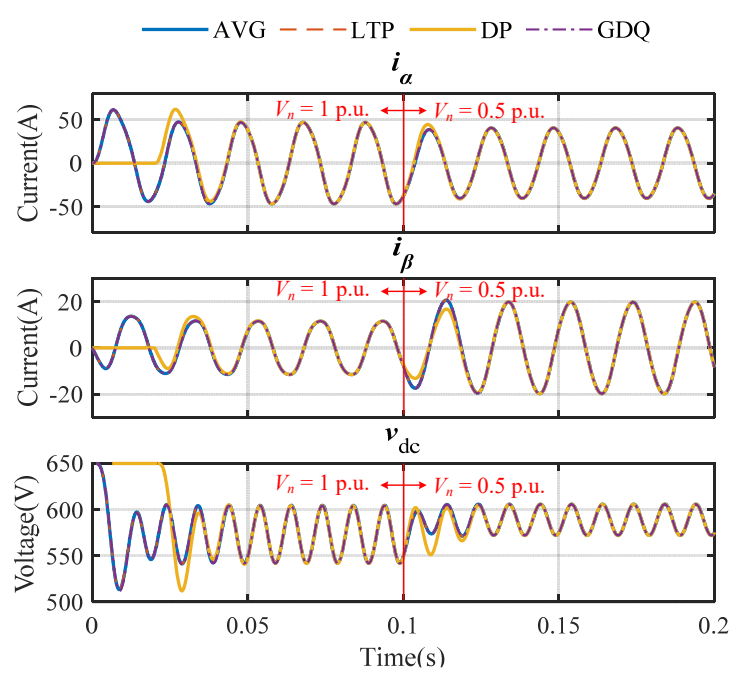

Fig. 8 Open-loop validation between different models on a three-phase converter in unbalanced grids.

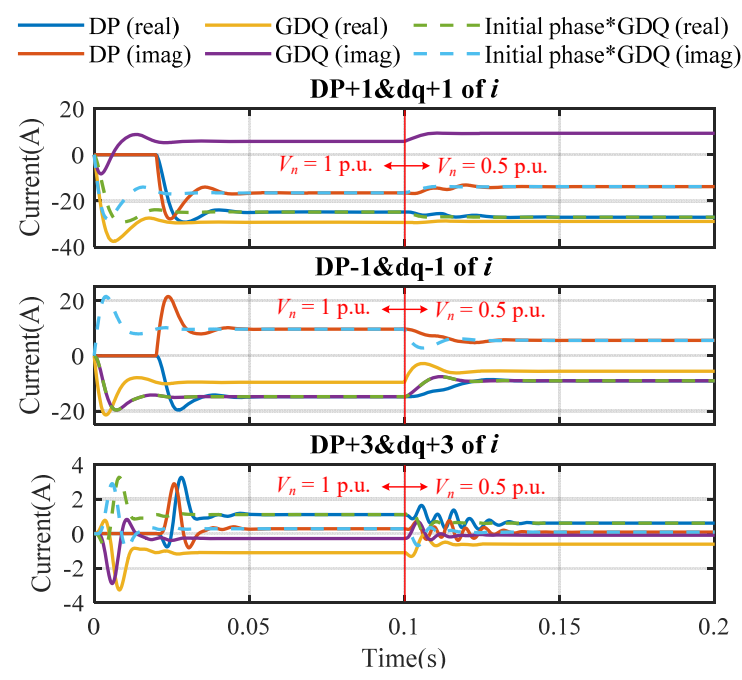

Fig. 9 Mathematical relationship validation between the DP and GDQ models.

way, the HTFs of different subsystems can be derived explicitly as given in Part $B$ of the Appendix, which form the closed-loop model of the entire converter system as Fig. 11. From the derived HTFs in the Appendix, it can be found that all the HTFs impacted by steady-state trajectories are defined based on $d q$-frame or dc variables (e.g., $\mathcal{D}_{d q}, I_{d q}, \mathcal{V}_{d}^{c}, \mathcal{V}_{\mathrm{dc}}$ ). Consequently, considering the major harmonic orders of 0 and \pm 2 is sufficient to modeling the converter system under unbalanced grid conditions.

The closed-loop ac admittance model can be derived as

$$
\left[\begin{array}{c}
\mathbf{I}_{\alpha \beta}\left(s-2 j \omega_{s}\right) \\
\mathbf{I}_{\alpha \beta}(s) \\
\mathbf{I}_{\alpha \beta}\left(s+2 j \omega_{s}\right) \\
\mathbf{I}_{\alpha \beta}^{*}\left(s-4 j \omega_{s}\right) \\
\mathbf{I}_{\alpha \beta}^{*}\left(s-2 j \omega_{s}\right) \\
\mathbf{I}_{\alpha \beta}^{*}(s)
\end{array}\right]=\boldsymbol{Y}_{\mathrm{ac}}\left[\begin{array}{c}
\mathbf{V}_{\alpha \beta}\left(s-2 j \omega_{s}\right) \\
\mathbf{V}_{\alpha \beta}(s) \\
\mathbf{V}_{\alpha \beta}\left(s+2 j \omega_{s}\right) \\
\mathbf{V}_{\alpha \beta}^{*}\left(s-4 j \omega_{s}\right) \\
\mathbf{V}_{\alpha \beta}^{*}\left(s-2 j \omega_{s}\right) \\
\mathbf{V}_{\alpha \beta}^{*}(s)
\end{array}\right],
$$

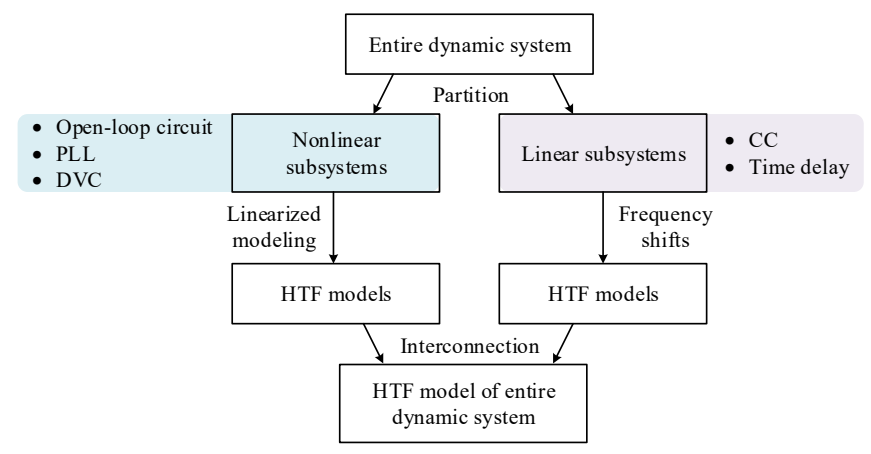

Fig. 10 Modular modeling procedures for a dynamic system.

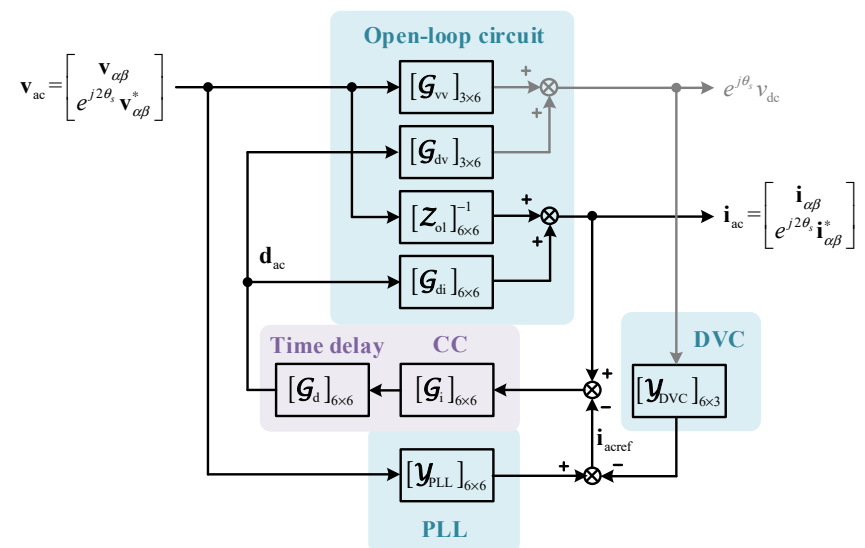

Fig. 11 Closed-loop model of the converter under unbalanced grid condition in complex $\alpha \beta$ frame.
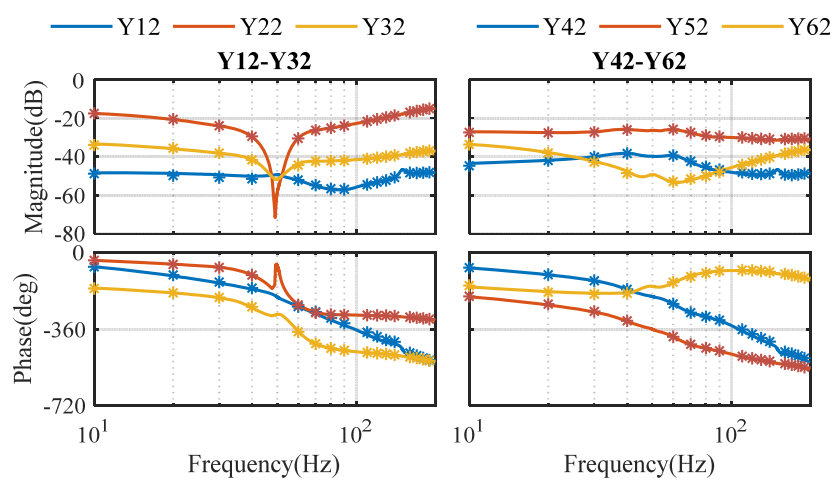

Fig. 12 Frequency-domain validation by admittance measurement of the converter.

where $\boldsymbol{Y}_{\text {ac }}$ is a 6-by-6 HTF given by

$$
\begin{aligned}
\boldsymbol{Y}_{\mathrm{ac}}= & {\left[\mathbf{I}-\boldsymbol{G}_{\mathrm{di}}\left(\mathbf{I}-\boldsymbol{G}_{\mathrm{d}} \boldsymbol{G}_{\mathrm{i}} \boldsymbol{\mathcal { V }}_{\mathrm{DVC}} \boldsymbol{G}_{\mathrm{dv}}\right)^{-1} \boldsymbol{G}_{\mathrm{d}} \boldsymbol{G}_{\mathrm{i}}\right]^{-1} } \\
& {\left[\boldsymbol{Z}_{\mathrm{ol}}^{-1}-\boldsymbol{G}_{\mathrm{di}}\left(\mathbf{I}-\boldsymbol{G}_{\mathrm{d}} \boldsymbol{G}_{\mathrm{i}} \boldsymbol{\mathcal { Y }}_{\mathrm{DVC}} \boldsymbol{G}_{\mathrm{dv}}\right)^{-1} \boldsymbol{G}_{\mathrm{d}} \boldsymbol{G}_{\mathrm{i}}\left(\boldsymbol{Y}_{\mathrm{PLL}}-\boldsymbol{\mathcal { Y }}_{\mathrm{DVC}} \boldsymbol{G}_{\mathrm{vv}}\right)\right] }
\end{aligned}
$$

To validate the frequency-domain model of the closed-loop converter system, the ac admittance is measured by frequency scan in simulation. The perturbation at $\omega$ for $\mathbf{v}_{\alpha \beta}$ is injected into the input voltage of the converters, which allows for measuring the frequency responses of the second-column of

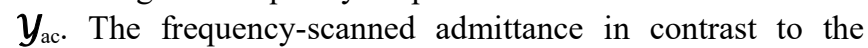
analytical model is shown in Fig. 12. The asterisks are the 


\section{IEEE REGULAR PAPER}

measured results and the solid lines are the analytical models. The good agreement verifies the accuracy of the frequencydomain modeling.

\section{CONCLUSION}

The mathematical bases and relationships of the HSS, DP and GDQ modeling methods have been rigorously proved in this work. The three methods share a common mathematical basis, by using the time-invariant representation of ac systems based on an orthogonal basis. Considering their mathematical relationships, the three methods yield a unified HTF model in the frequency domain:

1) For the HSS modeling, the unified HTF model can be derived from the real-valued LTP model first, then transformed through an extended complex transformation.

2) For the DP modeling, the complex/extended complex transformation and GA yield the DP model, which can be derived further as the unified HTF model through the Laplace transformation. The complex or extended complex transformation can be considered in any step.

3) For the GDQ modeling, it can also be applied to both real-valued or complex-valued LTP models. The complex-valued GDQ model can be further derived as the unified HTF model considering the initial phase rotation and the Laplace transformation.

The mathematical relationships of these models have been verified in the time domain on an open-loop converter system in unbalanced grid conditions, and the unified closed-loop frequency-domain model in the complex $\alpha \beta$ frame has been verified by frequency scan.

\section{APPENDIX}

\section{A. Open-Loop Modeling}

1) Nonlinear time-periodic model:

a) Real $\alpha \beta$-frame model:

$$
\begin{aligned}
& {\left[\begin{array}{l}
v_{\alpha} \\
v_{\beta}
\end{array}\right]=L \frac{\mathrm{d}}{\mathrm{d} t}\left[\begin{array}{l}
i_{\alpha} \\
i_{\beta}
\end{array}\right]+v_{\mathrm{dc}}\left[\begin{array}{l}
d_{\alpha} \\
d_{\beta}
\end{array}\right],} \\
& C \frac{\mathrm{d} v_{\mathrm{dc}}}{\mathrm{d} t}=\left[\begin{array}{ll}
d_{\alpha} & d_{\beta}
\end{array}\right]\left[\begin{array}{l}
i_{\alpha} \\
i_{\beta}
\end{array}\right]
\end{aligned}
$$

b) Complex $\alpha \beta$-frame model:

$$
\begin{aligned}
& {\left[\begin{array}{c}
\mathbf{v}_{\alpha \beta} \\
\mathbf{v}_{\alpha \beta}^{*}
\end{array}\right]=L \frac{\mathrm{d}}{\mathrm{d} t}\left[\begin{array}{l}
\mathbf{i}_{\alpha \beta} \\
\mathbf{i}_{\alpha \beta}^{*}
\end{array}\right]+v_{\mathrm{dc}}\left[\begin{array}{c}
\mathbf{d}_{\alpha \beta} \\
\mathbf{d}_{\alpha \beta}^{*}
\end{array}\right]}
\end{aligned}
$$

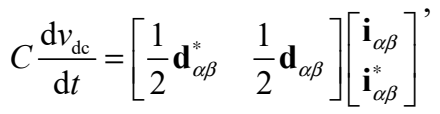

2) LTP model:

a) Real $\alpha \beta$-frame model:

$$
\begin{aligned}
& {\left[\begin{array}{l}
v_{\alpha} \\
v_{\beta}
\end{array}\right]=L \frac{\mathrm{d}}{\mathrm{d} t}\left[\begin{array}{l}
i_{\alpha} \\
i_{\beta}
\end{array}\right]+V_{\mathrm{dc}}(t)\left[\begin{array}{l}
d_{\alpha} \\
d_{\beta}
\end{array}\right]+\left[\begin{array}{c}
D_{\alpha}(t) \\
D_{\beta}(t)
\end{array}\right] v_{\mathrm{dc}},} \\
& C \frac{\mathrm{d} v_{\mathrm{dc}}}{\mathrm{d} t}=D_{\alpha}(t) i_{\alpha}+I_{\alpha}(t) d_{\alpha}+D_{\beta}(t) i_{\beta}+I_{\beta}(t) d_{\beta}
\end{aligned}
$$

where the steady-state trajectories are determined by $\alpha \beta$-frame variables $\left(D_{\alpha}(t), D_{\beta}(t), I_{\alpha}(t), I_{\beta}(t)\right)$ and $V_{\mathrm{dc}}(t)$.

b) Complex $\alpha \beta$-frame model:

$$
\begin{aligned}
& {\left[\begin{array}{c}
\mathbf{v}_{\alpha \beta} \\
\mathbf{v}_{\alpha \beta}^{*}
\end{array}\right]=L \frac{\mathrm{d}}{\mathrm{d} t}\left[\begin{array}{l}
\mathbf{i}_{\alpha \beta} \\
\mathbf{i}_{\alpha \beta}^{*}
\end{array}\right]+V_{\mathrm{dc}}(t)\left[\begin{array}{l}
\mathbf{d}_{\alpha \beta} \\
\mathbf{d}_{\alpha \beta}^{*}
\end{array}\right]+\left[\begin{array}{l}
\mathbf{D}_{\alpha \beta}(t) \\
\mathbf{D}_{\alpha \beta}^{*}(t)
\end{array}\right] v_{\mathrm{dc}}} \\
& C \frac{\mathrm{d} v_{\mathrm{dc}}}{\mathrm{d} t}=\frac{1}{2}\left(\begin{array}{l}
\mathbf{D}_{\alpha \beta}(t) \mathbf{i}_{\alpha \beta}^{*}+\mathbf{D}_{\alpha \beta}^{*}(t) \mathbf{i}_{\alpha \beta} \\
+\mathbf{I}_{\alpha \beta}^{*}(t) \mathbf{d}_{\alpha \beta}+\mathbf{I}_{\alpha \beta}(t) \mathbf{d}_{\alpha \beta}^{*}
\end{array}\right)
\end{aligned}
$$

where the steady-state trajectories are determined by $\alpha \beta$-frame variables $\left(\mathbf{D}_{\alpha \beta}(t), \mathbf{D}_{\alpha \beta}^{*}(t), \mathbf{I}_{\alpha \beta}(t), \mathbf{I}_{\alpha \beta}^{*}(t)\right)$ and $V_{\mathrm{dc}}(t)$.

c) Complex $\alpha \beta$-frame model considering ac- and dc-side frequency couplings:

$$
\begin{aligned}
& {\left[\begin{array}{c}
\mathbf{v}_{\alpha \beta} \\
e^{j 2 \theta_{s}} \mathbf{v}_{\alpha \beta}^{*}
\end{array}\right]=\left[\begin{array}{cc}
L \frac{\mathrm{d}}{\mathrm{d} t} & \\
& e^{j 2 \theta_{s}} L \frac{\mathrm{d}}{\mathrm{d} t} e^{-j 2 \theta_{s}}
\end{array}\right]\left[\begin{array}{c}
\mathbf{i}_{\alpha \beta} \\
e^{j 2 \theta_{s}} \mathbf{i}_{\alpha \beta}^{*}
\end{array}\right]} \\
& +V_{\mathrm{dc}}(t)\left[\begin{array}{c}
\mathbf{d}_{\alpha \beta} \\
e^{j 2 \theta_{s}} \mathbf{d}_{\alpha \beta}^{*}
\end{array}\right]+\left[\begin{array}{c}
\mathbf{D}_{d q}(t) \\
\mathbf{D}_{d q}^{*}(t)
\end{array}\right] e^{j \theta_{s}} v_{\mathrm{dc}}
\end{aligned}
$$

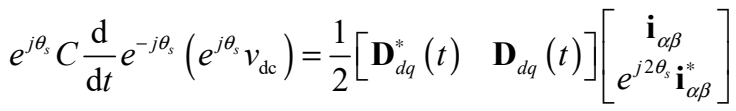

$$
\begin{aligned}
& +\frac{1}{2}\left[\begin{array}{ll}
\mathbf{I}_{d q}^{*}(t) & \mathbf{I}_{d q}(t)
\end{array}\right]\left[\begin{array}{c}
\mathbf{d}_{\alpha \beta} \\
e^{j 2 \theta_{s}} \mathbf{d}_{\alpha \beta}^{*}
\end{array}\right]
\end{aligned}
$$

where all the steady-state trajectories are determined by dqframe variables $\left(\mathbf{D}_{d q}(t), \mathbf{D}_{d q}^{*}(t), \mathbf{I}_{d q}(t), \mathbf{I}_{d q}^{*}(t)\right)$ and $V_{\mathrm{dc}}(t)$.

3) HTF model:

a) Based on Fourier coefficients of real $\alpha \beta$-frame variables:

$$
\begin{aligned}
& {\left[\begin{array}{l}
\nu_{\alpha}(s) \\
\nu_{\beta}(s)
\end{array}\right]=\mathcal{Z}_{L}\left[\begin{array}{l}
i_{\alpha}(s) \\
i_{\beta}(s)
\end{array}\right]+\mathcal{V}_{\mathrm{dc}}\left[\begin{array}{l}
\boldsymbol{d}_{\alpha}(s) \\
\boldsymbol{d}_{\beta}(s)
\end{array}\right]+\left[\begin{array}{l}
\mathcal{D}_{\alpha} \\
\mathcal{D}_{\beta}
\end{array}\right] \boldsymbol{\nu}_{\mathrm{dc}}(s)} \\
& \mathcal{Y}_{C} \boldsymbol{\nu}_{\mathrm{dc}}(s)=\mathcal{D}_{\alpha} \boldsymbol{i}_{\alpha}(s)+\mathcal{I}_{\alpha} \boldsymbol{d}_{\alpha}(s)+\mathcal{D}_{\beta} \boldsymbol{i}_{\beta}(s)+\mathcal{I}_{\beta} \boldsymbol{d}_{\beta}(s)
\end{aligned}
$$

$$
\text { where } v_{\alpha}(s)=\left[\begin{array}{c}
\vdots \\
V_{\alpha,-k}(s) \\
\vdots \\
V_{\alpha, 0}(s) \\
\vdots \\
V_{\alpha, k}(s) \\
\vdots
\end{array}\right]=\left[\begin{array}{c}
\vdots \\
V_{\alpha}\left(s-j k \omega_{1}\right) \\
\vdots \\
V_{\alpha}(s) \\
\vdots \\
V_{\alpha}\left(s+j k \omega_{1}\right) \\
\vdots
\end{array}\right] \text {, }
$$

and similar for other variables

$$
\begin{aligned}
& \mathcal{Z}_{L}=\operatorname{diag}\left\{\cdots,\left(s-j k \omega_{s}\right) L, \cdots, s L, \cdots,\left(s+j k \omega_{s}\right) L, \cdots\right\} \\
& \mathcal{Y}_{C}=\operatorname{diag}\left\{\cdots,\left(s-j k \omega_{s}\right) C, \cdots, s C, \cdots,\left(s+j k \omega_{s}\right) C, \cdots\right\} \\
& \mathcal{V}_{\mathrm{dc}}, \mathcal{D}_{\alpha}, \mathcal{D}_{\beta}, I_{\alpha}, I_{\beta} \text { are Toeplitz matrices }
\end{aligned}
$$

b) Based on Fourier coefficients of complex $\alpha \beta$-frame variables (equivalent to applying the transformation shown in Fig. 2 to (A3a)): 


$$
\begin{aligned}
{\left[\begin{array}{c}
v_{\alpha \beta}(s) \\
v_{\alpha \beta}^{*}(s)
\end{array}\right]=} & Z_{L}\left[\begin{array}{l}
i_{\alpha \beta}(s) \\
i_{\alpha \beta}^{*}(s)
\end{array}\right]+\mathcal{V}_{\mathrm{dc}}\left[\begin{array}{l}
d_{\alpha \beta}(s) \\
d_{\alpha \beta}^{*}(s)
\end{array}\right]+\left[\begin{array}{l}
\mathcal{D}_{\alpha \beta} \\
\mathcal{D}_{\alpha \beta}^{*}
\end{array}\right] \nu_{\mathrm{dc}}(s) \\
\mathcal{Y}_{C} \nu_{\mathrm{dc}}(s)= & \frac{1}{2} \mathcal{D}_{\alpha \beta} i_{\alpha \beta}^{*}(s)+\frac{1}{2} \mathcal{D}_{\alpha \beta}^{*} i_{\alpha \beta}(s) \\
& +\frac{1}{2} I_{\alpha \beta}^{*} d_{\alpha \beta}(s)+\frac{1}{2} I_{\alpha \beta} d_{\alpha \beta}^{*}(s)
\end{aligned}
$$

where $v_{\alpha \beta}(s)=v_{\alpha}(s)+j v_{\beta}(s), v_{\alpha \beta}^{*}(s)=\nu_{\alpha}(s)-j v_{\beta}(s)$ $\mathcal{D}_{\alpha \beta} \triangleq \mathcal{D}_{\alpha}+j \mathcal{D}_{\beta}, \mathcal{D}_{\alpha \beta}^{*} \triangleq \mathcal{D}_{\alpha}-j \mathcal{D}_{\beta}$

and similar for other variables

c) Reformulating signals considering frequency couplings (corresponding to the LTP model shown in (A2c)):

$$
\begin{array}{r}
{\left[\begin{array}{c}
v_{\alpha \beta}(s) \\
v_{\alpha \beta}^{*}\left(s-j 2 \omega_{s}\right)
\end{array}\right]=\left[\begin{array}{cc}
Z_{L}(s) & \\
& Z_{L}\left(s-j 2 \omega_{s}\right)
\end{array}\right]\left[\begin{array}{c}
i_{\alpha \beta}(s) \\
i_{\alpha \beta}^{*}\left(s-j 2 \omega_{s}\right)
\end{array}\right]} \\
+\mathcal{V}_{\mathrm{dc}}\left[\begin{array}{c}
d_{\alpha \beta}(s) \\
d_{\alpha \beta}^{*}\left(s-j 2 \omega_{s}\right)
\end{array}\right]+\left[\begin{array}{c}
\mathcal{D}_{d q} \\
\mathcal{D}_{d q}^{*}
\end{array}\right] \nu_{\mathrm{dc}}\left(s-j \omega_{s}\right) \\
\mathcal{Y}_{C}\left(s-j \omega_{s}\right) \nu_{\mathrm{dc}}\left(s-j \omega_{s}\right)=\frac{1}{2} \mathcal{D}_{d q} i_{\alpha \beta}^{*}\left(s-j 2 \omega_{s}\right)+\frac{1}{2} \mathcal{D}_{d q}^{*} i_{\alpha \beta}(s) \\
+\frac{1}{2} I_{d q}^{*} \boldsymbol{d}_{\alpha \beta}(s)+\frac{1}{2} I_{d q} d_{\alpha \beta}^{*}\left(s-j 2 \omega_{s}\right)
\end{array}
$$

where $v_{\alpha \beta}(s)$ is the same as shown in (A3b),

$\nu_{\alpha \beta}^{*}\left(s-j 2 \omega_{s}\right)$ and $\nu_{\mathrm{dc}}\left(s-j \omega_{s}\right)$ are frequency-shifted

$\mathcal{D}_{d q} \triangleq \mathcal{D}_{d}+j \mathcal{D}_{q}, \mathcal{D}_{d q}^{*} \triangleq \mathcal{D}_{d}-j \mathcal{D}_{q}$

and similar for other variables

4) DP models:

a) Based on real $\alpha \beta$-frame LTP model:

$\left[\begin{array}{l}\left\langle v_{\alpha}\right\rangle \\ \left\langle v_{\beta}\right\rangle\end{array}\right]=L \frac{\mathrm{d}}{\mathrm{d} t}\left[\begin{array}{l}\left\langle i_{\alpha}\right\rangle \\ \left\langle i_{\beta}\right\rangle\end{array}\right]+L \mathcal{N}\left[\begin{array}{l}\left\langle i_{\alpha}\right\rangle \\ \left\langle i_{\beta}\right\rangle\end{array}\right]+\mathcal{V}_{\mathrm{dc}}\left[\begin{array}{c}\left\langle d_{\alpha}\right\rangle \\ \left\langle d_{\beta}\right\rangle\end{array}\right]+\left[\begin{array}{c}\mathcal{D}_{\alpha} \\ \mathcal{D}_{\beta}\end{array}\right]\left\langle v_{\mathrm{dc}}\right\rangle,$,
$C \frac{\mathrm{d}}{\mathrm{d} t}\left\langle v_{\mathrm{dc}}\right\rangle+C \mathcal{N}\left\langle v_{\mathrm{dc}}\right\rangle=\mathcal{D}_{\alpha}\left\langle i_{\alpha}\right\rangle+I_{\alpha}\left\langle d_{\alpha}\right\rangle+\mathcal{D}_{\beta}\left\langle i_{\beta}\right\rangle+I_{\beta}\left\langle d_{\beta}\right\rangle$

where $\left\langle v_{\alpha}\right\rangle=\left[\begin{array}{c}\vdots \\ \left\langle v_{\alpha}\right\rangle_{-k} \\ \vdots \\ \left\langle v_{\alpha}\right\rangle_{0} \\ \vdots \\ \left\langle v_{\alpha}\right\rangle_{+k} \\ \vdots\end{array}\right]$, and similar for other variables

$\mathcal{N}=\operatorname{diag}\left\{\cdots,-j k \omega_{s}, \cdots, 0, \cdots, j k \omega_{s}, \cdots\right\}$

$\mathcal{V}_{\mathrm{dc}}, \mathcal{D}_{\alpha}, \mathcal{D}_{\beta}, I_{\alpha}, I_{\beta}$ are Toeplitz matrices

b) Based on complex $\alpha \beta$-frame LTP model (equivalent to applying the transformation shown in Fig. 2 to (A4a)):

$$
\begin{aligned}
& {\left[\begin{array}{c}
\left\langle\mathbf{v}_{\alpha \beta}\right\rangle \\
\left\langle\mathbf{v}_{\alpha \beta}^{*}\right\rangle
\end{array}\right]=L \frac{\mathrm{d}}{\mathrm{d} t}\left[\begin{array}{c}
\left\langle\mathbf{i}_{\alpha \beta}\right\rangle \\
\left\langle\mathbf{i}_{\alpha \beta}^{*}\right\rangle
\end{array}\right]+L \mathcal{N}\left[\begin{array}{l}
\left\langle\mathbf{i}_{\alpha \beta}\right\rangle \\
\left\langle\mathbf{i}_{\alpha \beta}^{*}\right\rangle
\end{array}\right]} \\
& +\mathcal{V}_{\mathrm{dc}}\left[\begin{array}{l}
\left\langle\mathbf{d}_{\alpha \beta}\right\rangle \\
\left\langle\mathbf{d}_{\alpha \beta}^{*}\right\rangle
\end{array}\right]+\left[\begin{array}{l}
\mathcal{D}_{\alpha \beta} \\
\mathcal{D}_{\alpha \beta}^{*}
\end{array}\right]\left\langle v_{\mathrm{dc}}\right\rangle \\
& C \frac{\mathrm{d}}{\mathrm{d} t}\left\langle v_{\mathrm{dc}}\right\rangle+C \mathcal{N}\left\langle v_{\mathrm{dc}}\right\rangle=\frac{1}{2} \mathcal{D}_{\alpha \beta}\left\langle\mathbf{i}_{\alpha \beta}^{*}\right\rangle+\frac{1}{2} I_{\alpha \beta}\left\langle\mathbf{d}_{\alpha \beta}^{*}\right\rangle \\
& +\frac{1}{2} \mathcal{D}_{\alpha \beta}^{*}\left\langle\mathbf{i}_{\alpha \beta}\right\rangle+\frac{1}{2} I_{\alpha \beta}^{*}\left\langle\mathbf{d}_{\alpha \beta}\right\rangle
\end{aligned}
$$

where $\left\langle\mathbf{v}_{\alpha \beta}\right\rangle=\left[\begin{array}{c}\vdots \\ \left\langle\mathbf{v}_{\alpha \beta}\right\rangle_{-k} \\ \vdots \\ \left\langle\mathbf{v}_{\alpha \beta}\right\rangle_{0} \\ \vdots \\ \left\langle\mathbf{v}_{\alpha \beta}\right\rangle_{+k} \\ \vdots\end{array}\right]=\left[\begin{array}{c}\vdots \\ \left\langle v_{\alpha}\right\rangle_{-k}+j\left\langle v_{\beta}\right\rangle_{-k} \\ \vdots \\ \left\langle v_{\alpha}\right\rangle_{0}+j\left\langle v_{\beta}\right\rangle_{0} \\ \vdots \\ \left\langle v_{\alpha}\right\rangle_{+k}+j\left\langle v_{\beta}\right\rangle_{+k} \\ \vdots \\ \vdots \\ \vdots \\ \left\langle\mathbf{v}_{\alpha \beta}^{*}\right\rangle=\left[\begin{array}{c}\left.\mathbf{v}_{\alpha \beta}^{*}\right\rangle_{-k} \\ \vdots \\ \left\langle\mathbf{v}_{\alpha \beta}^{*}\right\rangle_{0} \\ \vdots \\ \left\langle\mathbf{v}_{\alpha \beta}^{*}\right\rangle_{+k} \\ \vdots\end{array}\right]=\left[\begin{array}{c}\left\langle v_{\alpha}\right\rangle_{-k}-j\left\langle v_{\beta}\right\rangle_{-k} \\ \vdots \\ \left\langle v_{\alpha}\right\rangle_{0}-j\left\langle v_{\beta}\right\rangle_{0} \\ \vdots \\ \left\langle v_{\alpha}\right\rangle_{+k}-j\left\langle v_{\beta}\right\rangle_{+k} \\ \vdots\end{array}\right], \text { such that }\end{array}\right.$

$\left\langle\mathbf{v}_{\alpha \beta}^{*}\right\rangle_{+k}=\left(\left\langle\mathbf{v}_{\alpha \beta}\right\rangle_{-k}\right)^{*}$ and similar for other variables

$\mathcal{N}=\operatorname{diag}\left\{\cdots,-j k \omega_{s}, \cdots, 0, \cdots, j k \omega_{s}, \cdots\right\}$

$\mathcal{V}_{\mathrm{dc}}, \mathcal{D}_{\alpha \beta}, \mathcal{D}_{\alpha \beta}^{*}, \mathcal{D}_{z}, I_{\alpha \beta}, I_{\alpha \beta}^{*}, I_{z}$ are Toeplitz matrices

5) Complex-valued GDQ model (based on dominant harmonic components):

$$
\begin{aligned}
& {\left[\begin{array}{c}
\overline{\mathbf{v}}_{d q-1} \\
\overline{\mathbf{v}}_{d q+1} \\
\overline{\mathbf{v}}_{d q+3}
\end{array}\right]=L \frac{\mathrm{d}}{\mathrm{d} t}\left[\begin{array}{c}
\overline{\mathbf{i}}_{d q-1} \\
\overline{\mathbf{i}}_{q q+1} \\
\overline{\mathbf{i}}_{d q+3}
\end{array}\right]+L N\left[\begin{array}{c}
\overline{\mathbf{i}}_{d q-1} \\
\overline{\mathbf{i}}_{d q+1} \\
\overline{\mathbf{i}}_{d q+3}
\end{array}\right]} \\
& +Q \mathcal{V}_{\mathrm{dc}} Q^{-1}\left[\begin{array}{l}
\overline{\mathbf{d}}_{d q-1} \\
\overline{\mathbf{d}}_{d q+1} \\
\overline{\mathbf{d}}_{d q+3}
\end{array}\right]+Q e^{-j \varphi_{p}} D_{\alpha \beta} Q^{-1} \underbrace{Q\left\langle v_{\mathrm{dc}}\right\rangle}_{\mathbf{v}_{\mathrm{dc}}} \\
& {\left[\begin{array}{c}
\overline{\mathbf{v}}_{d q-1}^{*} \\
\overline{\mathbf{v}}_{d q+1}^{*} \\
\overline{\mathbf{v}}_{d q+3}^{*}
\end{array}\right]=L \frac{\mathrm{d}}{\mathrm{d} t}\left[\begin{array}{c}
\overline{\mathbf{i}}_{d q-1}^{*} \\
\overline{\mathbf{i}}_{d q+1}^{*} \\
\overline{\mathbf{d}}_{d q+3}^{*}
\end{array}\right]-L N\left[\begin{array}{c}
\overline{\mathbf{i}}_{d q-1}^{*} \\
\overline{\mathbf{i}}_{d q+1}^{*} \\
\overline{\mathbf{i}}_{d q+3}^{*}
\end{array}\right]} \\
& +Q^{-1} \mathcal{V}_{\mathrm{dc}}^{*} Q\left[\begin{array}{l}
\overline{\mathbf{d}}_{d q-1}^{*} \\
\overline{\mathbf{d}}_{d q+1}^{*} \\
\overline{\mathbf{d}}_{d q+3}^{*}
\end{array}\right]+Q^{-1} e^{j \varphi_{p}} D_{\alpha \beta}^{*} Q^{-1} \underbrace{Q\left\langle v_{\mathrm{dc}}\right\rangle}_{\mathbf{v}_{\mathrm{dc}}}
\end{aligned}
$$




$$
\begin{aligned}
& C \frac{\mathrm{d}}{\mathrm{d} t} \underbrace{Q\left\langle v_{\mathrm{dc}}\right\rangle}_{\mathbf{v}_{\mathrm{dc}}}+C N \underbrace{Q\left\langle v_{\mathrm{dc}}\right\rangle}_{\mathbf{v}_{\mathrm{dc}}}=\frac{1}{2} Q D_{\alpha \beta} e^{-j \varphi_{n}} Q^{-1}\left[\begin{array}{c}
\overline{\mathbf{i}}_{d q-1}^{*} \\
\overline{\mathbf{i}}_{d q+1}^{*} \\
\overline{\mathbf{i}}_{d q+3}^{*}
\end{array}\right] \\
& +\frac{1}{2} Q I_{\alpha \beta} e^{-j \varphi_{n}} Q^{-1}\left[\begin{array}{l}
\overline{\mathbf{d}}_{d q-1}^{*} \\
\overline{\mathbf{d}}_{d q+1}^{*} \\
\overline{\mathbf{d}}_{d q+3}^{*}
\end{array}\right] \\
& +\frac{1}{2} Q D_{\alpha \beta}^{*} e^{j \varphi_{p}} Q^{-1}\left[\begin{array}{c}
\overline{\mathbf{i}}_{d q-1} \\
\overline{\mathbf{i}}_{d q+1} \\
\overline{\mathbf{i}}_{d q+3}
\end{array}\right] \\
& +\frac{1}{2} Q I_{\alpha \beta}^{*} e^{j \varphi_{p}} Q^{-1}\left[\begin{array}{l}
\overline{\mathbf{d}}_{d q-1} \\
\overline{\mathbf{d}}_{d q+1} \\
\overline{\mathbf{d}}_{d q+3}
\end{array}\right]
\end{aligned}
$$

where $\overline{\mathbf{v}}_{d q+3}=\frac{1}{T} \int_{t-T}^{t} \mathbf{v}_{\alpha \beta} e^{-j\left(2 \theta_{p}-\theta_{n}\right)} d \tau=e^{-j\left(2 \varphi_{p}-\varphi_{n}\right)}\left\langle\mathbf{v}_{\alpha \beta}\right\rangle_{+3}$

$\overline{\mathbf{v}}_{d q+1}=\frac{1}{T} \int_{t-T}^{t} \mathbf{v}_{\alpha \beta} e^{-j \theta_{p}} d \tau=e^{-j \varphi_{p}}\left\langle\mathbf{v}_{\alpha \beta}\right\rangle_{+1}$

$\overline{\mathbf{v}}_{d q-1}=\frac{1}{T} \int_{t-T}^{t} \mathbf{v}_{\alpha \beta} e^{-j \theta_{n}} d \tau=e^{-j \varphi_{n}}\left\langle\mathbf{v}_{\alpha \beta}\right\rangle_{-1}$

$N=\operatorname{diag}\left\{-j \omega_{s}, j \omega_{s}, j 3 \omega_{s}\right\}$

$D_{\alpha \beta}=\left[\begin{array}{ccc}\left\langle D_{\alpha \beta}\right\rangle_{+1} & \left\langle D_{\alpha \beta}\right\rangle_{-1} & 0 \\ \left\langle D_{\alpha \beta}\right\rangle_{+3} & \left\langle D_{\alpha \beta}\right\rangle_{+1} & \left\langle D_{\alpha \beta}\right\rangle_{-1} \\ 0 & \left\langle D_{\alpha \beta}\right\rangle_{+3} & \left\langle D_{\alpha \beta}\right\rangle_{+1}\end{array}\right]$

$D_{\alpha \beta}^{*}=\left[\begin{array}{ccc}0 & \left\langle D_{\alpha \beta}^{*}\right\rangle_{+1} & \left\langle D_{\alpha \beta}^{*}\right\rangle_{-1} \\ \left\langle D_{\alpha \beta}^{*}\right\rangle_{+1} & \left\langle D_{\alpha \beta}^{*}\right\rangle_{-1} & \left\langle D_{\alpha \beta}^{*}\right\rangle_{-3} \\ \left\langle D_{\alpha \beta}^{*}\right\rangle_{-1} & \left\langle D_{\alpha \beta}^{*}\right\rangle_{-3} & 0\end{array}\right]$

$Q=\left[\begin{array}{ccc}e^{j\left(\varphi_{p}-\varphi_{n}\right)} & & \\ & 1 & \\ & & e^{-j\left(\varphi_{p}-\varphi_{n}\right)}\end{array}\right]$

$\mathbf{v}_{\mathrm{dc}}=Q\left\langle v_{\mathrm{dc}}\right\rangle=\left[\begin{array}{lll}e^{j\left(\varphi_{p}-\varphi_{n}\right)} & & \\ & 1 & \\ & & e^{-j\left(\varphi_{p}-\varphi_{n}\right)}\end{array}\right]\left[\begin{array}{c}\left\langle v_{\mathrm{dc}}\right\rangle_{-2} \\ \left\langle v_{\mathrm{dc}}\right\rangle_{0} \\ \left\langle v_{\mathrm{dc}}\right\rangle_{+2}\end{array}\right] \triangleq\left[\begin{array}{c}v_{\mathrm{dc}-2} \\ v_{\mathrm{dc} 0} \\ v_{\mathrm{dc}+2}\end{array}\right]$

and similar for other variables

B. Closed-Loop Modeling

1) Open-loop HTFs

$$
\begin{gathered}
\boldsymbol{Z}_{\mathrm{ol}}=\boldsymbol{Z}_{\mathrm{ac}}+\left[\begin{array}{l}
\mathcal{D}_{d q} \\
\mathcal{D}_{d q}^{*}
\end{array}\right] \frac{\boldsymbol{Z}_{\mathrm{dc}}\left(s-j \omega_{s}\right)}{2}\left[\begin{array}{ll}
\mathcal{D}_{d q}^{*} & \mathcal{D}_{d q}
\end{array}\right], \\
\mathcal{G}_{\mathrm{di}}=-\boldsymbol{Z}_{\mathrm{ol}}^{-1}\left(\left[\begin{array}{ll}
\mathcal{V}_{\mathrm{dc}} & \\
& \mathcal{V}_{\mathrm{dc}}
\end{array}\right]+\left[\begin{array}{l}
\mathcal{D}_{d q} \\
\mathcal{D}_{d q}^{*}
\end{array}\right] \frac{\boldsymbol{Z}_{\mathrm{dc}}\left(s-j \omega_{s}\right)}{2}\left[\begin{array}{ll}
I_{d q}^{*} & I_{d q}
\end{array}\right]\right),
\end{gathered}
$$

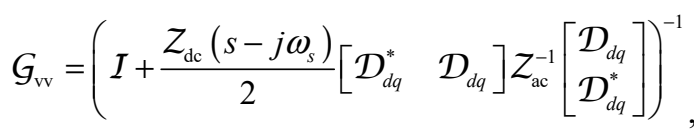

$$
\begin{aligned}
& \cdot \frac{Z_{\mathrm{dc}}\left(s-j \omega_{s}\right)}{2}\left[\mathcal{D}_{d q}^{*} \quad \mathcal{D}_{d q}\right] Z_{\mathrm{ac}}^{-1} \\
& G_{\mathrm{dv}}=\left(I+\frac{Z_{\mathrm{dc}}\left(s-j \omega_{s}\right)}{2}\left[\mathcal{D}_{d q}^{*} \quad \mathcal{D}_{d q}\right] Z_{\mathrm{ac}}^{-1}\left[\begin{array}{c}
\mathcal{D}_{d q} \\
\mathcal{D}_{d q}^{*}
\end{array}\right]\right)^{-1} \\
& \frac{Z_{\mathrm{dc}}\left(s-j \omega_{s}\right)}{2}\left(\left[\begin{array}{ll}
I_{d q}^{*} & I_{d q}
\end{array}\right]-\left[\begin{array}{ll}
\mathcal{D}_{d q}^{*} & \mathcal{D}_{d q}
\end{array}\right] Z_{\mathrm{ac}}^{-1}\left[\begin{array}{ll}
\mathcal{V}_{\mathrm{dc}} & \\
& \mathcal{V}_{\mathrm{dc}}
\end{array}\right]\right) \\
& \text { where } Z_{\mathrm{ac}}=\left[\begin{array}{ll}
Z_{\mathrm{L}}(s) & \\
& Z_{\mathrm{L}}\left(s-j 2 \omega_{s}\right)
\end{array}\right] \text {, } \\
& Z_{\mathrm{L}}(s)=\left[\begin{array}{lll}
\left(s-j 2 \omega_{s}\right) L & & \\
& s L & \\
& & \left(s+j 2 \omega_{s}\right) L
\end{array}\right] \text {, } \\
& Z_{\mathrm{dc}}(s)=\left[\begin{array}{lll}
\frac{1}{\left(s-j 2 \omega_{s}\right) C} & & \\
& \frac{1}{s C} & \\
& & \frac{1}{\left(s+j 2 \omega_{s}\right) C}
\end{array}\right],
\end{aligned}
$$

$\mathcal{V}_{\mathrm{dc}}, \mathcal{D}_{d q}, I_{d q}$ are 3-by-3 HTFs obtained by steady-state trajectories.

2) PLL HTF

$$
\begin{gathered}
\mathcal{Y}_{\mathrm{PLL}}=\frac{1}{2}\left[\begin{array}{cc}
I_{d q \text { ref }} \mathcal{T}_{\Delta \theta} \mathcal{G}_{\mathrm{PLL}}\left(s-j \omega_{s}\right) \mathcal{T}_{\Delta \theta}^{*} & -I_{\text {dqref }} \mathcal{T}_{\Delta \theta} \mathcal{G}_{\mathrm{PLL}}\left(s-j \omega_{s}\right) \mathcal{T}_{\Delta \theta} \\
-I_{\text {dqref }}^{*} \mathcal{T}_{\Delta \theta}^{*} \mathcal{G}_{\mathrm{PLL}}\left(s-j \omega_{s}\right) \mathcal{T}_{\Delta \theta}^{*} & I_{d q r e f}^{*} \mathcal{T}_{\Delta \theta}^{*} \mathcal{G}_{\mathrm{PLL}}\left(s-j \omega_{s}\right) \mathcal{T}_{\Delta \theta}
\end{array}\right], \\
\text { where } \mathcal{G}_{\mathrm{PLL}}(s)=\left(I+\mathcal{G}_{\text {Notch-PI-I }}(s) \mathcal{V}_{d}^{c}\right)^{-1} \mathcal{G}_{\text {Notch-PI-I }}(s), \\
G_{\text {Notch-PI-I }}=\operatorname{diag}\left\{\begin{array}{l}
G_{\text {Notch-PI-I }}\left(s-j 2 \omega_{s}\right), G_{\text {Notch-PI-I }}(s) \\
G_{\text {Notch-PI-I }}\left(s+j 2 \omega_{s}\right)
\end{array}\right\} \text { and }
\end{gathered}
$$

$G_{\text {Notch-PI-I }}(s)$ is the transfer function of the PLL including notch filter, PI controller and integrator.

$\mathcal{T}_{\Delta \theta}$ is the HTF of $e^{j \Delta \theta}$, where $\Delta \theta$ is the steady-state phase difference between the PLL control dq frame and the positivesequence system dq frame [17]. $\mathcal{V}_{d}^{c}$ is the HTF of $V_{d}^{c}(t)$, which is the steady-state $d$-axis voltage in PLL control $d q$ frame. $I_{d q r e f}$ is the HTF of the steady-state $I_{d q \text { ref }}(t)$.

3) DVC HTF

$$
\mathcal{Y}_{\mathrm{DVC}}=\left[\begin{array}{c}
\mathcal{T}_{\Delta \theta} \mathcal{G}_{\mathrm{DVC}}\left(s-j \omega_{s}\right) \\
\mathcal{T}_{\Delta \theta} \mathcal{G}_{\mathrm{DVC}}\left(s-j \omega_{s}\right)
\end{array}\right],
$$

where

$\boldsymbol{G}_{\mathrm{DVC}}(s)=\operatorname{diag}\left\{G_{\mathrm{dvc}}\left(s-j 2 \omega_{s}\right), G_{\mathrm{dvc}}(s), G_{\mathrm{dvc}}\left(s+j 2 \omega_{s}\right)\right\}$ and $G_{\text {dvc }}(s)$ is the transfer function of the DVC PI controller.

4) $\mathrm{CC}$ and time delay HTFs

$$
\mathcal{G}_{\mathrm{i}}=\frac{1}{V_{\mathrm{dc}}}\left[\begin{array}{ll}
\mathcal{G}_{\mathrm{CC}}(s) & \\
& \mathcal{G}_{\mathrm{CC}}\left(s-2 j \omega_{s}\right)
\end{array}\right],
$$


where $G_{\mathrm{CC}}=\operatorname{diag}\left\{G_{i}\left(s-j 2 \omega_{s}\right), G_{i}(s), G_{i}\left(s+j 2 \omega_{s}\right)\right\} \quad$ and $G_{i}(s)$ is the transfer function of the CC PR controller.

$$
\mathcal{G}_{\mathrm{d}}=\left[\begin{array}{ll}
\mathcal{G}_{\text {delay }}(s) & \\
& \mathcal{G}_{\text {delay }}\left(s-2 j \omega_{s}\right)
\end{array}\right],
$$

where $G_{\text {delay }}=\operatorname{diag}\left\{G_{d}\left(s-j 2 \omega_{s}\right), G_{d}(s), G_{d}\left(s+j 2 \omega_{s}\right)\right\}$ and

$G_{d}(s)$ is the transfer function of the time delay.

\section{REFERENCES}

[1] X. Wang and F. Blaabjerg, "Harmonic Stability in Power Electronic Based Power Systems: Concept, Modeling, and Analysis," IEEE Trans. Smart Grid, vol. 10, no. 3, pp. 2858-2870, May 2019.

[2] G. W. Wester and R. D. Middlebrook, "Low-frequency characterization of switched dc-dc converters," IEEE Trans. Aero. Electron. Syst., vol. AES-9, pp. 376-385, May 1973.

[3] R. D. Middlebrook and S. Cuk, "A general unified approach to modeling switching converter power stages," in Proc. IEEE Power Electron. Spec. Conf., 1976, pp. 18-34.

[4] S. Hiti, D. Boroyevich, and C. Cuadros, "Small-signal modeling and control of three-phase PWM converters," in Proc. IEEE Ind. Appl. Society Annual Meet., Denver, CO, Oct. 1994, pp. 1143-1150.

[5] L. Harnefors, M. Bongiorno, and S. Lundberg, "Input-admittance calculation and shaping for controlled voltage-source converters," IEEE Trans. Ind. Electron., vol. 54, no. 6, pp. 3323-3334, Nov. 2007.

[6] B. Wen, D. Boroyevich, R. Burgos, P. Mattavelli, and Z. Shen, "Analysis of D-Q small-signal impedance of grid-tied inverters," IEEE Trans. Power Electron., vol. 31, no. 1, pp. 675-687, Jan. 2016.

[7] L. Harnefors, "Modeling of three-phase dynamic systems using complex transfer functions and transfer matrices," IEEE Trans. Ind. Electron., vol. 54, no. 4, pp. 2239-2248, Aug. 2007.

[8] Y. Gu, Y. Li, Y. Zhu and T. Green, "Impedance-Based Whole-System Modeling for a Composite Grid via Embedding of Frame Dynamics," IEEE Trans. Power Syst., Early Access, doi: 10.1109/TPWRS.2020.3004377.

[9] A. Rygg, M. Molinas, C. Zhang, and X. Cai, "A modified sequencedomain definition and its equivalence to dq-domain impedance definition for the stability analysis of ac power electronic systems," IEEE J. of Emerg. Sel. Topics Power Electron., vol. 4, no. 4, pp. 13831396, Dec. 2016.

[10] C. T. Rim, "Unified General Phasor Transformation for AC Converters," IEEE Trans. Power Electron., vol. 26, no. 9, pp. 2465-2475, Sept. 2011.

[11] X. Wang, L. Harnefors, and F. Blaabjerg, "Unified impedance model of grid-connected voltage-source converters," IEEE Trans. Power Electron., vol. 33, no. 2, pp. 1775-1787, Feb. 2018.

[12] Y. Liao and X. Wang, "Stationary-frame complex-valued frequencydomain modeling of three-phase power converters," IEEE J. Emerg. Sel. Topics Power Electron., vol. 8, no. 2, pp. 1922-1933, June 2020.

[13] E. Mollerstedt and B. Bernhardsson, "Out of control because of harmonics-an analysis of the harmonic response of an inverter locomotive," IEEE Control Syst. Mag., vol. 20, no. 4, pp. 70-81, Aug. 2000.

[14] T. Noda, A. Semlyen and R. Iravani, "Harmonic domain dynamic transfer function of a nonlinear time-periodic network," IEEE Trans. Power Del., vol. 18, no. 4, pp. 1433-1441, Oct. 2003.

[15] J. Lyu, X. Zhang, X. Cai and M. Molinas, "Harmonic State-Space Based Small-Signal Impedance Modeling of a Modular Multilevel Converter with Consideration of Internal Harmonic Dynamics," IEEE Trans. Power Electron., vol. 34, no. 3, pp. 2134-2148, March 2019.

[16] H. Wu and X. Wang, "Dynamic Impact of Zero-Sequence Circulating Current on Modular Multilevel Converters: Complex-Valued AC Impedance Modeling and Analysis," IEEE J. Emerg. Sel. Topics Power Electron., vol. 8, no. 2, pp. 1947-1963, June 2020.

[17] Y. Liao, X. Wang, X. Yue and L. Harnefors, "Complex-Valued Multifrequency Admittance Model of Three-Phase VSCs in Unbalanced Grids," IEEE J. Emerg. Sel. Topics Power Electron., vol. 8, no. 2, pp. 1934-1946, June 2020.
[18] H. Yang, H. Just, M. Eggers and S. Dieckerhoff, "Linear Time-Periodic Theory Based Modeling and Stability Analysis of Voltage Source Converters," IEEE J. Emerg. Sel. Topics Power Electron., Early Access, doi: 10.1109/JESTPE.2020.3003379.

[19] P. C. Stefanov and A. M. Stankovic, "Modeling of UPFC operation under unbalanced conditions with dynamic phasors," IEEE Trans. Power Syst., vol. 17, no. 2, pp. 395-403, May 2002.

[20] J. J. Rico, M. Madrigal and E. Acha, "Dynamic harmonic evolution using the extended harmonic domain," IEEE Trans. Power Del., vol. 18, no. 2, pp. 587-594, April 2003.

[21] O. C. Sakinci and J. Beerten, "Generalized dynamic phasor modeling of the MMC for small-signal stability analysis," IEEE Trans. Power Del., vol. 34, no. 3, pp. 991-1000, June 2019.

[22] P. J. Hart, J. Goldman, R. H. Lasseter and T. M. Jahns, "Impact of Harmonics and Unbalance on the Dynamics of Grid-Forming, Frequency-Droop-Controlled Inverters," IEEE J. Emerg. Sel. Topics Power Electron., vol. 8, no. 2, pp. 976-990, June 2020.

[23] P. Rioual, H. Pouliquen and J. -. Louis, "Regulation of a PWM rectifier in the unbalanced network state using a generalized model," IEEE Trans. Power Electron., vol. 11, no. 3, pp. 495-502, May 1996.

[24] A. Jamshidifar and D. Jovcic, "Small-Signal Dynamic DQ Model of Modular Multilevel Converter for System Studies," IEEE Trans. Power Del., vol. 31, no. 1, pp. 191-199, Feb. 2016.

[25] T. Li, A. M. Gole and C. Zhao, "Harmonic Instability in MMC-HVDC Converters Resulting From Internal Dynamics," IEEE Trans. Power Del., vol. 31, no. 4, pp. 1738-1747, Aug. 2016.

[26] N. M. Wereley, "Analysis and control of linear periodically time varying systems," Ph.D. dissertation, Dept. of Aeronautics and Astronautics, MIT, 1991.

[27] A. Packard, K. Poolla, and R. Horowitz, "Dynamic systems and feedback class notes,” Dept. Mech. Eng., Univ. California, Berkeley, CA, USA, Spring 2005.

[28] J. Sun, "Small-Signal Methods for AC Distributed Power Systems-A Review," IEEE Trans. Power Electron., vol. 24, no. 11, pp. 2545-2554, Nov. 2009.

[29] H. Sandberg, E. Mollerstedt and Bernhardsson, "Frequency-domain analysis of linear time-periodic systems," IEEE Trans. Auto. Cont., vol. 50, no. 12, pp. 1971-1983, Dec. 2005.

[30] Y. Li, Y. Gu and T. C. Green, "Interpreting Frame Transformations in AC Systems as Diagonalization of Harmonic Transfer Functions," IEEE Trans. Circ. Syst. I: Reg. Papers, vol. 67, no. 7, pp. 2481-2491, July 2020.

[31] S. R. Sanders, J. M. Noworolski, X. Z. Liu and G. C. Verghese, "Generalized averaging method for power conversion circuits," IEEE Trans. Power Electron., vol. 6, no. 2, pp. 251-259, Apr. 1991.

[32] D. Maksimovic, A. M. Stankovic, V. J. Thottuvelil and G. C. Verghese, "Modeling and simulation of power electronic converters," in Proceedings of the IEEE, vol. 89, no. 6, pp. 898-912, June 2001, doi: $10.1109 / 5.931486$

[33] X. Yue, X. Wang, and F. Blaabjerg, "Review of small-signal modeling methods including frequency-coupling dynamics of power converters," IEEE Trans. Power Electron., vol. 34, no. 4, pp. 3313-3328, Apr. 2019.

[34] S. D. Sudhoff, "Multiple reference frame analysis of an unsymmetrical induction machine," IEEE Trans. Energy Conv., vol. 8, no. 3, pp. 425432, Sept. 1993

[35] J. E. Ormrod, "Analysis and control of linear periodically time varying systems," M.E. thesis, Electrical and Computer Engineering, University of Canterbury, 2013.

[36] J. Kwon, X. Wang, F. Blaabjerg, C. L. Bak, A. R. Wood and N. R. Watson, "Linearized Modeling Methods of AC-DC Converters for an Accurate Frequency Response," IEEE J. Emerg. Sel. Topics Power Electron., vol. 5, no. 4, pp. 1526-1541, Dec. 2017.

[37] P. D. Rua, O. C. Sakinci, and J. Beerten, "Comparative Study of Dynamic Phasor and Harmonic State-Space Modeling for Small-Signal Stability Analysis," Elect. Power Syst. Research, vol. 189, no. 106626, pp. $1-8$, Dec. 2020

[38] O. C. Sakinci and J. Beerten, "Equivalent Multiple dq-Frame Model of the MMC using Dynamic Phasor Theory in the $\alpha \beta z$-Frame," IEEE Trans. Power Del., Early Access, Sep. 2020, doi: 10.1109/TPWRD.2020.3025388. 


\section{IEEE REGULAR PAPER}

[39] Z. Xu, B. Li, S. Zhang, L. Han, J. Hu and D. Xu, "Study on equivalence of MMC modeling under multi-dq frames and harmonic state space," in Proc. 8th Renew. Power Gen. Conf., Shanghai, China, 2019, pp. 1-6.

[40] M. H. Hirsch, S. Smale, and R. L. Devaney, Differential Equations, Dynamical Systems \& an Introduction to Chaos, 3rd ed., Elsevier, 2013.

[41] M. Reed and B. Simon, Methods of Modern Mathematical Physics: Functional Analysis, Academic Press, 1972.

[42] G. C. Paap, "Symmetrical components in the time domain and their application to power network calculations," IEEE Trans. Power Syst., vol. 15 , no. 2 , pp. $522-528$, May. 2000

[43] J. M. Undrill and T. E. Kostyniak, "Subsynchronous oscillations, part 1 - comprehensive system stability analysis," IEEE Trans. Power Appar. Syst., vol. PAS-95, no. 4, pp. 1446-1455, Jul./Aug. 1976.

[44] C. Spanias and I. Lestas, "A System Reference Frame Approach for Stability Analysis and Control of Power Grids," IEEE Trans. Power Syst., vol. 34, no. 2, pp. 1105-1115, Mar. 2019.

[45] F. Cavazzana, A. Khodamoradi, H. Abedini, and P. Mattavelli, "Analysis of an impedance modeling approach for droop-controlled inverters in system DQ frame," in Proc. IEEE Energy Conversion Congress and Exposition (ECCE), Baltimore, MD, USA, Sep. 2019.

[46] S. Wang, Z. Liu, J. Liu, D. Boroyevich, and R. Burgos, "Small-signal modeling and stability prediction of parallel droop-controlled inverters based on terminal characteristics of individual inverters," IEEE Trans. Power Electron., vol. 35, no. 1, pp. 1045-1063, Jan. 2020.

[47] Y. Yin, R. Zane, J. Glaser and R. W. Erickson, "Small-signal analysis of frequency-controlled electronic ballasts," IEEE Trans. Circ. and Syst. I: Fund. Theory and Appl., vol. 50, no. 8, pp. 1103-1110, Aug. 2003.

[48] J. Groves, "Small-signal analysis using harmonic balance methods," in Proc. IEEE Power Electron. Spec. Annual Conf. (PESC), Cambridge, MA, USA, 1991, pp. 74-79. 